\title{
ADP-ribosylating adjuvant reveals plasticity in CDC1 cells that drive mucosal Th17 development and protection against influenza virus infection
}

Nils Lycke ( $\square$ nils.lycke@microbio.gu.se)

University of Gothenburg https://orcid.org/0000-0003-1155-4861

Mohammad Arabpour

University of Gothenburg

Cristina Lebrero-Fernández

University of Gothenburg https://orcid.org/0000-0002-9024-1240

Karin Schön

Univ. of Gothenburg

Anneli Stromberg

University of Gothenburg

Vanja Börjesson

University of Gothenburg

Katherina Lahl

Lund University

\section{Davide Angeletti}

University of Gothenburg https://orcid.org/0000-0002-5256-1972

William Agace

Technical University of Denmark

\section{Article}

Keywords: CTA1-DD, Th17 cells, adjuvant, cholera toxin , CD103, cDC1 cells, Batf3-/- mice, influenza infection

Posted Date: August 26th, 2021

DOI: https://doi.org/10.21203/rs.3.rs-801483/v1

License: (1) (1) This work is licensed under a Creative Commons Attribution 4.0 International License.

Read Full License 


\section{ADP-ribosylating adjuvant reveals plasticity in cDC1 cells \\ that drive mucosal Th17 development and protection against influenza virus infection}

Mohammad Arabpour $^{1}$, Cristina Lebrero ${ }^{1}$, Karin Schön ${ }^{1}$, Anneli Strömberg ${ }^{1}$, Vanja Börjesson ${ }^{2}$ Katharina Lahl ${ }^{3}$, Davide Angeletti ${ }^{1}$, William Agace ${ }^{3}$ and Nils Lycke ${ }^{*}$

${ }^{1}$ MIVAC-Mucosal Immunobiology \& Vaccine Center, Department of Microbiology and Immunology, Institute of Biomedicine, University of Gothenburg, Gothenburg, Sweden,

${ }^{2}$ Bioinformatics Core facility, The Sahlgrenska Academy, University of Gothenburg, Gothenburg, Sweden

${ }^{3}$ Immunology Section, Lund University, BMC D14, 221-84 Lund, Sweden

${ }^{4}$ Mucosal Immunology group, Department of Health Technology, Technical University of Denmark, Kemitorvet, 2800 Kgs. Lyngby, Denmark

* Corresponding author

Running title: $\mathrm{cDC} 1$ cells promote Th17 differentiation

Keywords: CTA1-DD, Th17 cells, adjuvant, cholera toxin , CD103, cDC1 cells, Batf3-/- mice, influenza infection

Conflict of Interest: The authors declare no competing financial interest 


\section{Abstract}

Migratory dendritic cells carrying CD103 are the targets for mucosal vaccines. These belong to either of two lineage restricted subsets, $\mathrm{CDC} 1$ or cDC2 cells, which have been linked to priming of functionally distinct CD4 T cells. However, recent studies have identified plasticity in cDC2 cells with overlapping functions with $\mathrm{cDC} 1$ cells, while the converse has not been reported. We genetically engineered a vaccine adjuvant platform that targeted the cholera toxin A1 (CTA1) ADP-ribosylating enzyme to $\mathrm{CD}_{103^{+}} \mathrm{cDC} 1$ and $\mathrm{cDC} 2$ cells using a single chain antibody (scFv) to CD103. Unexpectedly, intranasal immunization with the CTA1-svFcCD103 adjuvant modified cDC1 cells to effectively prime Th17 cells, a function normally restricted to cDC2 cells. In fact, cDC2 cells were dispensible. Single cell RNAseq analysis revealed upregulation of Th17-promoting gene signatures in sorted cDC1 cells. Our finding represents a major shift of paradigm as we have documented functional plasticity in cDC1 cells. 


\section{Introduction}

Migratory dendritic cells (DCs) play a key role as antigen-presenting cells (APC) for priming of CD4 and CD8 T cells in the draining lymph nodes (Durai and Murphy 2016). These cells are CD11 $\mathrm{c}^{\text {high }}$ and $\mathrm{MHCII}{ }^{\text {high }}$ and migrate from the mucosal membrane to the draining lymph node after taking up antigen (Steinman et al. 1979). In this way antigen recognition following nasal immunization is mediated by migratory DCs that present processed peptides on MHC I or II molecules to naïve $\mathrm{T}$ cells in the $\mathrm{T}$ cell zone of the mediastinal lymph node (mLN) (Lycke 2012) (Bernasconi et al. 2020). On the other hand, in steady state migratory DCs help maintain tolerance via induction of regulatory T cells (Tregs) in the draining lymph node (Vander Lugt et al. 2017). Three main populations of migratory DCs have been identified and these belong to the classical DCs (cDCs), which are phenotypically distinct and have been linked to unique APC-functions (Anderson et al. 2020; Guilliams et al. 2014; Heath and Carbone 2009). Differential surface expression of CD103 and CD11b provide the basis for lineage separation

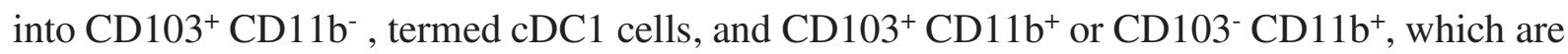
cDC2 double (cDC2DP) or single positive (cDC2SP) cells, respectively. The development of cDC1 and cDC2 cells is strictly controlled by gene expression as pre-DCs develop into these subsets in the bone marrow and they appear to be stable end-stages of differentiation with distinct transcriptional profiles (Anderson, Murphy, and Briseno 2018) (Schlitzer et al. 2015; Brown et al. 2019; Cabeza-Cabrerizo et al. 2019; See et al. 2017; Dutertre et al. 2019). The cDC1 cells, expressing Irf8, Batf3 , Id2 and Nfil3 genes, are known to cross-present MHC Irestricted peptides and, hence, stimulate CD8 T cells, but also CD4 T cell priming is effective as Th1 responses are induced (Ferris et al. 2020; Tussiwand et al. 2015; Dudziak et al. 2007). The cDC2 cells, expressing Irf4, Sirp $\alpha$ and Zeb2 genes, on the other hand, can be divided into Klf4- or Notch2-dependent functional subsets and these prime CD4 T cells and promote Th2 and Tfh/Th17 differentiation, respectively (Tussiwand et al. 2015; Schlitzer et al. 2015; Murphy et al. 2016; Briseno et al. 2018; Dutertre et al. 2019). Thus, immune responses against infection can be dependent on access to a specific cDC-subset in the tissues and, for example, protection 
against Toxoplasma gondii or Schistosoma manosni parasite infections require cDC1 and cDC2 cells, respectively (Mashayekhi et al. 2011; Mayer et al. 2017). Moreover, studies of protection against $C$. rodentium showed that $\mathrm{cDC} 2$ cells are required for IL-23 production whereas this function could not be replaced by $\mathrm{cDC} 1$ cells, supporting the notion that $\mathrm{cDC} 1$ cells are largely non-reduntant with cDC2 cells (Satpathy et al. 2013). On the other hand tissue localization and environmental cues may define cDC-subset functions, because, for example, Th2 responses were stimulated by cDC2DP cells in the small intestine, while this was done by cDC2SP cells in the colon (Mayer et al. 2017; Brown et al. 2019). A recent study indicated that cDC2 subsets can change APC-function following adjuvant modulation (Lee, Zhang, et al. 2020). It was demonstrated that cAMP-inducing agents, such as cholera toxin (CT) adjuvant, caused plasticity in Klf4-dependent cDC2 cells by repressing Irf4-expression, leading to a Th17- rather than Th2-promoting activity, a function normally associated with Notch2-expressing cDC2DP cells (Lee, Zhang, et al. 2020; Bedoui and Heath 2015; Tussiwand et al. 2015). Moreover, cDC2 cells expressing Tbet have been reported to support Th1 cell development, which speaks in favor of functional plasticity in the cDC2 subset (Brown et al. 2019). By contrast, functional plasticity was not observed with $\mathrm{cDC} 1$ cells following exposure to cAMP-inducing substances (Lee, Zhang, et al. 2020). Thus, our current understanding of migratory cDCs is that they exhibit strict lineage commitment, but it appears that functional plasticity exist only among cDC2 cells, whereas it has not been described for $\mathrm{CDC} 1$ cells. So , for example, no study has demonstrated that $\mathrm{CDC} 1$ cells can induce Th17 responses.

To improve vaccine efficacy different strategies have been employed and efforts have focused on targeting vaccines to DCs (Tacken et al. 2007). The targeting has been achieved in many different ways, employing specific targeting elements, such as lectins or specific monoclonal antibodies (Mabs), or different antigen formulations, of which nanoparticles are a good example (Pati, Shevtsov, and Sonawane 2018; Perciani et al. 2020). Whereas, the nanoparticles most often do not secure binding to a specific subset of DCs, conjugation of antigens to anti-CD103 or DEC205- Mabs can achieve selective targeting to cDC-subsets (Semmrich et al. 2012; Bernasconi et al. 2020; Boscardin et al. 2006; Cruz et al. 2014). However, to be effective at 
priming of $\mathrm{T}$ cells most of the targeting strategies have required complementing toll like receptor (TLR)-ligand or anti-CD40 Mab stimulation (Macri et al. 2016). Hence, targeting of vaccines to cDC cells has not been found sufficient for improving vaccine efficacy without employing added adjuvant capacity to the formulation.

We have previously developed a fusion protein that accommodates the adjuvant activity of the enzymatically active CTA1-subunit of CT and a dimer of a D-targeting moiety, derived from Staphylococcus aureus proteinA (Agren, Ekman, et al. 1999). This fusion protein exerts comparable adjuvant effects to those found with intact CT, however, without any of the many toxic side effects of CT (Lycke and Lebrero-Fernandez 2018). In particular when given intranasally (i.n) CTA1-DD strongly augments cell-mediated as well as humoral immune responses (Lycke 2012). Antigenic epitopes coupled or genetically fused to the CTA1-DD fusion protein or simply admixed with the adjuvant acquire strongly enhanced immunogenicity (Schussek et al. 2020). For example, the CTA1-3M2e-DD fusion protein, which carries the influenza M2e peptide, when given i.n, stimulates strong heterosubtypic protection against influenza virus infection (Eliasson et al. 2018). The present study was undertaken to unravel how targeting of the adjuvant active CTA1-enzyme to $\mathrm{CD} 103^{+} \mathrm{DCs}$ could influence the immune response and which cDC subsets are responsible for the adjuvant effect. To this end we designed and expressed new fusion proteins with a single chain antibody specific for CD103 (scFvCD103) (Semmrich et al. 2012). The CTA1-scFvCD103 platform was then equipped with well defined peptides that were genetically fused into the molecule (CTA1-peptide-CD103 ) and used to analyze the peptide-specific $\mathrm{T}$ cell response. This way we can now provide compelling evidence that CTA1 targeted to $\mathrm{CD} 103^{+} \mathrm{cDCs}$ changes the function of $\mathrm{cDC} 1$ cells to effectively prime for Th17 responses. 


\section{Results}

\section{The CTA1-CD103 fusion protein binds specifically to $\mathrm{CD103}^{+}$dendritic cells}

To achieve targeting of the CTA1-adjuvant to cDCs we fused the CTA1-encoding gene to the scFvCD103 gene and also incorporated gene segments encoding the MHC class I and/or class II-restricted peptides from ovalbumin; the SIINFEKL (I)- and/or p323 (II)-peptides, respectively (Figure 1A-B) (Lycke \& Lebrero 2018). These constructs were then compared to our original CTA1-DD constructs, carrying the same inserted peptides, with regard to their ADP-ribosylating ability. We found that both the CTA1-II-CD103 and the CTA1-II-DD constructs exerted ADP-ribosylation, fully compatible to that of the CT-holotoxin (Figure 1C). As expected, the CTA1R9K-mutation in both constructs obliterated the enzymatic activity completely (Figure 1C) (Agren, Ekman, et al. 1999). The binding-specificity of the fusion proteins to $\mathrm{CD} 103^{+} \mathrm{DCs}$ was assessed by using flow cytometry of migratory DCs (CD11 $\mathrm{c}^{\text {high }}$ MHC II ${ }^{\text {high}}$ ) isolated from mesenteric lymph nodes (MLN) from wild-type (WT) or CD103-/- mice. Both -DD and -CD103 targeted constructs bound migratory DCs, but the CTA1-II-CD103 fusion protein failed to bind DCs from CD103-/- mice (Figure 1D, Supplementary figure 1). Of note, the CD103-constructs bound more effectively to CD103+ DCs than -DD constructs (Figure1D). Furthermore, freshly isolated DCs from the mediastinal lymph node (mLNs) demonstrated binding of -CD103 constructs to both cDC1 $\left(\mathrm{CD} 103^{+} \mathrm{CD} 11 \mathrm{~b}^{-}\right)$and $\mathrm{cDC} 2$ double positive $(\mathrm{cDC} 2 \mathrm{DP})\left(\mathrm{CD}_{103}{ }^{+} \mathrm{CD} 11 \mathrm{~b}^{+}\right)$subsets, while -DD constructs in addition bound $\mathrm{cDC} 2$ single positive (cDC2SP)(CD103- CD11 $^{+}$) cells (Figure1E). Again the CD103-targeted construct bound better to cDC1- than to cDC2DP cells and more effectively than -DD fusion proteins (Figure1E). No binding to B cells in the mLN was observed, demonstrating that these CTA1-adjuvants, indeed, targeted migratory cDCs and preferentially cDC1 cells (Figure1E).

CD8 $T$ cell responses and cytotoxicity are enhanced by the CD103-targeted CTA1adjuvant 
Because cDC1 cells $\left(\mathrm{CD} 103^{+} \mathrm{CD} 11 \mathrm{~b}^{-}\right)$are known to cross-present and prime MHC class Irestricted CD8 T cell responses we investigated whether the CTA1-I/II-CD103 and CTA1-I/IIDD fusion proteins could stimulate SIINFEKL-specific CD8 T cell responses (Anderson et al. 2020). To this end C57B16 mice (Ly5.2+) were adoptively transferred with OT I T cells $\left(\right.$ Ly5. $\left..1^{+}\right)$ that were labeled with CFSE prior to i.v injections. Firstly we identified to what extent targeted migratory $\mathrm{CD} 103^{+} \mathrm{CD}^{-11 b^{-}} \mathrm{DCs}$ in the $\mathrm{mLN}$ carried SIINFEKL-peptide following i.n immunizations. We found that CD103-targeting of the CTA1-adjuvant induced a 3-fold higher surface expression of the SIINFEKL/MHC I complex in cDC1 cells compared to that provided by DD-fusion proteins, as assessed by flow cytometry and a labelled SIINFEKL/MHC I complex-specific Mab (Figure 2A). Subsequently,we observed that priming of OT I cells was also more effective in the draining mLN following i.n immunizations with CD103- as compared to DD-constructs (Figure 2B). We found that $90 \%$ of the OT I cells were primed, whereas an equimolar dose of the DD-construct was less effective, albeit much more effective than an equimolar dose of ovalbumin (Figure 2B). The more effective priming of OT I cells resulted in higher frequencies of IFN $\gamma$-producing cells and enhanced cytotoxicity in vivo (Figure 2C-D). Intranasal immunizations with CTA1-II-CD103, without the SIINFEKL peptide, failed to stimulate cytotoxicity in OT I cells (Figure 2D).

\section{CD103-targeted CTA1-adjuvant augments protection against influenza virus infection}

Next we evaluated the adjuvant ability of the CTA1-enzyme when CD103- or DD-fusion proteins were used for i.n immunizations of Balb/c mice against influenza virus infections (Eliasson et al. 2008). We have previously established that i.n immunizations with the CTA1M2e-DD fusion protein effectively stimulates M2e-specific lung resident memory Th17 cells, which provided strong heterosubtypic protection against a live influenza virus challenge infection, while CTA1-DD adjuvant alone did not protect (Eliasson et al. 2018). To this end we developed a CTA1-M2e-CD103 fusion protein to compare the protective properties of CD103and DD-fusion proteins. We found that CD103-targeting was much superior to the DDconstructs in preventing a lethal influenza virus infection (Figure 3A). Indeed, while 100\% of 
the mice survived after i.n immunizations with CTA1-M2e-CD103 only $40 \%$ survived infection with the highly virulent A/PR/8/1934 (H1N1) strain (4xLD50 dose) in the CTA1-M2e-DD immunized group (Figure 3A). The stronger protection correlated with a higher frequency of M2e-tetramer binding Th17 cells (ror $\gamma \mathrm{t}^{+}$) in the lungs in CD103- compared to DD- fusion protein immunized and challenged mice (Figure 3B). As previously documented no M2especific CD8 T cells or Th1 cells were stimulated by the i.n immunization and a significant proportion of the M2e-specific Th17 cells were $\mathrm{CD} 69^{+}$, indicative of lung resident memory cells (Figure 3B) (Eliasson et al. 2008; Bernasconi et al. 2016). By contrast, no difference between specific serum anti-M2e IgG or local bronchioalveolar lavage (BAL) IgA antibody titers were detected in -CD103 and -DD- fusion protein immunized mice (Figure 3A). Thus, it appeared that CD103-targeting was more effective than DD-constructs at promoting M2especific lung Th17-responses, which was associated with better protection against a live influenza virus infection.

To extend the analysis we went on to compare the adjuvant efficacy of the -CD103-and -DDbased fusion proteins, using a fixed dose of soluble protein antigen, tetanus toxoid (TT) or NPchicken gammaglobulin (NP-CGG) and decreasing doses of admixed adjuvant. It was observed that CD103-targeted CTA1-adjuvant gave between 35-100-fold stronger specific antibody responses than -DD constructs and it was highly effective at doses as low as $0.014 \mu \mathrm{M}$ (Supplementary figure 2). Thus, CD103-targeting was significantly more efficacious at augmenting CTA1-mediated adjuvanticity than the DD-constructs.

Enhanced priming of Th17 cells in draining lymph nodes following i.n immunization with CD103-targeted fusion proteins

To follow up with a more detailed investigation of Th17-priming after i.n immunizations we employed ovalbumin-specific (p323) OT II TCR transgenic CD4 T cells in an adoptive transfer model (Gribonika et al. 2019). Similar to the M2e-specific CD4 T cell response, experiments with OT II cells confirmed that CD103-targeting of the CTA1-adjuvant effectively stimulated CD4 $\mathrm{T}$ cell proliferation in vivo (Figure 4A). As expected, an enzymatically inactive mutant 
CTA1-molecule (CTA1R9K-II-CD103) was ineffective at stimulating OT II cell proliferation, in agreement with our previous studies (Figure 4A) (Eliasson et al. 2018). Noteworthy, the CD103-targeting itself did not exert adjuvant functions, attesting to that it was the enzymatic activity of CTA1 that was crucial for adjuvanticity. Interestingly, CD103-targeted CTA1adjuvant was more effective at retaining a strong priming ability even at 4 days following immunization (Figure 4B). Furthermore, the CD103-targeted fusion protein effectively promoted a Th17 cell response in OT II cells in the $\mathrm{mLN}$, albeit Tbet $^{+}$Th1 cells were also induced (Figure 4C). Also Th2 and Tfh cells were induced but less frequent than in OVA-only or DD-construct immunized mice (Supplementary figure 3A-C). Roughly 65\% of the CD4 T cells were Th17 cells while Th1, Th2 and Tfh cells together comprised 35\% (Supplementary figure 3D). ELISPOT-detection of IL-17 spot-forming cells (SFC) or cytokine assessments in culture supernatants from isolated OT II cells from $\mathrm{mLN}$ of i.n immunized mice confirmed a strong IL-17A response, while IFN $\gamma$ producing cells were 3-fold lower, but still augmented compared to an equimolar dose of ovalbumin (Figure 4D, Supplementary figure 3C). A complementing gene expression analysis of isolated OT II cells from mLN of fusion protein i.n immunized mice attested to a prominent Th17 cell mRNA signature with upregulated expression of rorc, rora, $I L-17 a$, and $I L-22$ compared to that found with OT II cells from OVAonly immunized mice (Figure 4E) (Castro et al. 2017). Taken together, CD103-targeting of the CTA1-adjuvant favored Th17 cell differentiation in mLN following i.n immunizations.

\section{The CTA1-adjuvant is acting through $\mathrm{cDC} 1$ cells in CD103-targeted fusion proteins}

To address which subset of $\mathrm{CD} 103^{+} \mathrm{DCs}$, $\mathrm{CDC} 1$ or cDC2DP cells, that the CD103-targeted CTA1-adjuvant was acting through we undertook experiments in genetically defined mice lacking distinct cDC subsets. To this end we employed Batf3-/- mice, lacking cDC1 cells, and huCD207/DTA mice, which have been found to be deficient in cDC2DP cells in the small intestinal lamina propria (LP) and MLN (Theisen et al. 2018; Welty et al. 2013). In addition, CD103-/- and WT C57B1/6 mice were also included in the analysis. We confirmed that Batf3/- mice lack cDC1 cells in the mLN and the huCD207/DTA mice did not have cDC2DP cells 
in the migratory cDC populations at this inductive site (Figure 5A). Conversely, Batf3-/- mice had migratory cDC2DP cells and huCD207/DTA mice had intact $\mathrm{cDC} 1\left(\mathrm{XCR} 1^{+} \mathrm{CD} 11 \mathrm{~b}^{-}\right)$cells in the $\mathrm{mLN}$ (Figure 5A). Moreover, CD103-/- mice had cDC1 (XCR1+CD103-) cells in the mLN. The cDC2SP $\left(\mathrm{CD} 103^{-} \mathrm{CD} 11 \mathrm{~b}^{+}\right)$DC subset was present in all three mouse strains (Figure 5A). We predicted that if the CD103-targeted CTA1-adjuvant was dependent on the CCD1 cells it would not work in Batf3-/- nor in CD103-/- mice for priming of OT II T cells. Following a single i.n immunization after adoptive transfer of OT II CD4 T cells we found that, indeed, CD103-targeted CTA1-adjuvant failed to stimulate OT II T cells in Batf3-/- as well as in CD103-/- mice (Figure 5B). Whereas DD-constructs effectively primed OT II CD4 T cells in CD103-/- mice, they, unexpectedly, failed to do this in Batf3-/- mice, suggesting that also DDconstructs preferentially mediated their priming activity through $\mathrm{cDC} 1$ cells (Figure 5B). Moreover, both CTA1-adjuvants effectively primed OT II CD4 T cells in the absence of cDC2DP cells in the huCD207/DTA mice (Figure 5B). The dependence on cDC1 cells was further confirmed in bone marrow (BM) chimeric littermate WT mice (50\% Batf3-/- and 50\% CD103-/- or WT BM) that hosted both cDC1 and cDC2 cells, but lacked expression of CD103 on cDC1 cells (Figure 5B). In these mice DD-constructs were effective, while-CD103-targeted CTA1-adjuvant failed to function (Figure 5B). With regard to cross-presentation we found that, priming of OT I CD8 T cells was lost in Batf3-/- mice with both CD103- and DD-targeted SIINFEKL-carrying constructs (Figure 5C). Taken together, these experiments clearly demonstrated that the targeted CTA1-adjuvants mediated their enhanced priming effects on CD4 T cells through cDC1 cells, while cDC2DP or cDC2SP cells appeared to be dispensible.

\section{Isolated cDC1 cells from intranasally immunized mice effectively induce Th17 cell differentiation ex vivo}

Because of the outcome of these in vivo experiments we turned to more rigorously controlled in vitro experiments. Given that our results suggested that $\mathrm{CDC} 1$ cells were mediating the effect of CD103-targeted CTA1-adjuvant we asked if highly enriched migratory cDC1 cells from the 
mLN following i.n immunization could drive OT II CD4 $\mathrm{T}$ cell proliferation and Th17 differentiation in vitro (Figure 6A). Indeed, only cDC1 cells from immunized mice stimulated cell division (CFSE-dilution) and Th17 (ror $\left.\mathrm{t}^{+}\right)$cell differentiation, whereas cDC1 cells from unimmunized mice failed to do so (Figure 6B). Thus, only cDC1 cells exposed to the fusion protein in vivo could activate the OT II cells, strongly supporting the observations in WT and Batf3-/- mice that cDC1 cells are the critical mediators of Th17 responses in our model system. Importantly, we observed no Tbet ${ }^{+}$OT II CD4 T cells in the cultures and no IFN $\gamma$ was produced (Figure 6C). Rather, enriched cDC1 cells from i.n CTA1-II-CD103 immunized mice stimulated significant levels of IL-17, IL-21 and IL-22, indicative of Th17 cells, as well as IL-1 $\beta$, IL-6 and IL-23, cytokines that drive Th17 development (Figure 6D). In vitro cultures enriched for cDC1 cells from mLN from unimmunized mice developed ror $\gamma \mathrm{t}^{+}$OT II CD4 T cells only when CTA1-II-CD103 fusion protein was added to the cultures (Supplementary figure 4). In addition, enriched cDC1 cells from mLN together with E $\alpha$-specific TCR Tg CD4 T cells cultured with E $\alpha-D D$ antigen and CTA1-II-CD103 or other adjuvants; Poly I:C, LPS and CpG, revealed that only the CTA1-adjuvant induced ror $\mathrm{t}^{+}$Th17 cells in vitro (Figure 6E) (Bernasconi et al. 2020). By contrast, the TLR-dependent adjuvants stimulated IFN $\gamma$ production, indicative of Th1 $\left(\right.$ Tbet $^{+}$) responses, as predicted (Figure 6E) (Cancel et al. 2019). CD4 T cells without co-culture with cDC1 cells failed to respond to the different stimuli (Supplementary figure 4). Thus, highly enriched cDC1 cells responded to the ADP-ribosylating CTA1 adjuvant with Th17 priming, while TLR-dependent adjuvants promoted Th1 and IFN $\gamma$-responses in vitro.

\section{Single cell RNAseq analysis reveals strong transcriptional impact on cDC1 cells of CTA1-} adjuvant

We investigated to what extent migratory DCs changed gene expression profiles as a consequence of CTA1-adjuvant targeting using the 10X Chromium platform and single cell RNAseq (scRNAseq) analysis (Becht et al. 2018). To this end C57Bl/6 mice received a single i.n dose of CTA1-II-CD103 or CTA1-II-DD and FACS-sorted migratory DCs in the mLN were 
subjected to scRNAseq analysis. The gene expression profiles were compared with those of migratory DCs from unimmunized PBS-treated control mice. To secure that we analyzed DCs that had bound the fusion protein we used fusion proteins that carried an E$\alpha$-peptide, so that we could identify the targeted cells as they expressed E $\alpha$-peptide/MHC II complexes using labelled

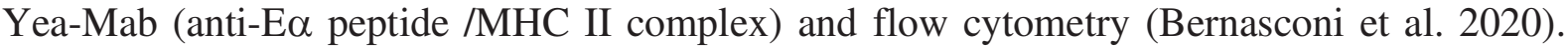
This way we exclusively analyzed migratory DCs targeted by CTA1-adjuvant in the mLN and compared their gene expression profiles with those obtained from cDCs from unimmunized PBS-treated control mice. Of note, the isolated control cDCs from unimmunized mice represent naturally activated migratory cells (Brown et al. 2019). After 24h following i.n administration of $50 \mu \mathrm{M}$ doses of CTA1-E $\alpha-C D 103$ or-DD we isolated and FACS-sorted Yea-Mab ${ }^{+}$DCs into high purity. After quality control and filtering we used the Seurat $\mathrm{R}$ toolkit to perform unsupervised clustering of the DCs based on differentially expressed genes (DEG) and the data was visualized using uniform manifold approximation and projection (UMAP) in two dimensions (Figure 7A-B) (Stuart et al. 2019). We analyzed a minimum of 500 cells in each category. The migratory DCs were distributed in 5 clusters and a distinct separation of cDC1 and $\mathrm{cDC} 2$ cells into these clusters was evident using published gene lists defining the subsets (Figure 7C, Supplementary figure 5A,B) (Brown et al. 2019). A striking observation was that migratory cDC1 cells underwent a more dramatic change following binding with CTA1adjuvant than the corresponding cDC2 cells (Figure 7A-C). Thus, both CD103- and DDtargeted cDC1 cells were found in clusters 3 and 4, while cDC1 cells from unimmunized mice were restricted to cluster 1 and 2 (Figure 7A-B). By contrast, cDC2 cells were found in cluster 0 and both CTA1-exposed and control migratory DCs inter-mingled in this cluster (Figure 7BC). Hence, the most dramatic shift occured in $\mathrm{cDC} 1$ cells, while $\mathrm{cDC} 2$ cells appeared less affected by the CTA1-adjuvant. The heat-map of the top 25 differentially regulated genes in migratory DCs from control or CTA1-treated mice also identified that cDC1 cells were the most affected, as evident in clusters 3 and 4 as opposed to gene expression in clusters 1 and 2 (Figure 7D). Even though -CD103 constructs were found to be more effective than DD-constructs at 
targeting cDC1 cells, the difference in gene expression profiles was minimal between the two with only $R g s-1, C d 83, \operatorname{Rel}$ and $C c r 7$ genes differentially expressed (Supplemental figure 5C). Nevertheless, both targeting constructs were effective at promoting CTA1-mediated immunomodulation of cDC1 cells, while cDC2 cells were less affected (Figure 7E). A set of the 10 most significantly DEG were identified in $\mathrm{cDC} 1$ cells and these genes were linked to activation and CD4 T cell priming functions (Figure 7E). Of note, Stat4, Scin and Cytip genes have all been associated with DC activation/migration and Fabp5 gene expression has linked fatty acid metabolism to Th17 differentiation (Lee, Kim, et al. 2020; Li et al. 2009).

We generated a pseudotime trajectory of gene expression in $\mathrm{CDC} 1$ cells using the Monocle 3 program (Figure 8A-C)(Cao et al. 2019). This analysis indicated that cDC1 cells had developed a gene expression profile that could represent a transition of function from the unmanipulated cDC1 cells to those exposed to the CTA1-adjuvant (cluster 3 and 4). (Figure 8A-D). Strikingly, when subsetting on $\mathrm{CDC} 1$ cells and applying a selection of critical genes known to be driving Th17 cell differentiation we observed a clear increase in such genes along the pseudotime trajectory (Figure 8E) (Hirota et al. 2012; Segura et al. 2013). Indeed, genes encoding costimulatory and Th17-promoting factors (IL-1b, IL-6, Tgfb1, Tgfb2, Tnfsf4 (Ox40L), Tnfrsf9 (CD137) IL-12b (p40), Timd4, Cd80, Cd86) were distinctly up-regulated in adjuvant exposed, as opposed to unimmunized control, cDC1 cells (Figure 8D-E, Supplemental figure 5D). An extended gene set enrichment analysis (GSEA) using a gene list taken from a publication of human inflammatory DCs, reported to promote Th17 responses, was performed with the list of co-differentially upregulated genes found in CTA1-exposed cCD1 cells (Segura et al. 2013). The GSEA showed significant enrichment of DEG that previously have been linked to Th17 promoting activity (Figure $8 \mathrm{~F}$ ). To further the analysis, the subsetted $\mathrm{cDC} 1$ population was analyzed for genes that change across the pseudotime. Genes were then grouped into modules using Louvain community analysis. Several activation and signalling pathways were involved as the $\mathrm{CDC} 1$ transitioned from the PBS cluster state to the most active CTA1-exposed cells (cluster 3) and this was seen with the modules showing most dramatic changes in gene expression (Figure 8G-H, Supplementary Excel files ). For example, modules hosting Stat3, 
Rel, Irf8, Crem and Stat4 and genes encoding activation, cell adhesion and co-stimulatory molecules (Anxa5, Bhlhe40, Cd86, Cd200, Cytip, Itga4, Flrt3, Scin, Tnfaip8, ox-40l) or genes involved in cell division, motility and survival (Cd81, Cd83, Ccr7, Map4k4) and cytokine and chemokine relevant genes (Anxa2, Il-12b, Map3k14) were significantly up-regulated in CTA1adjuvant exposed cDC1 cells (cluster 3 and 4), consistent with a strong CD4 T cell and Th17 priming function (Figure 8I). Furthermore, genes linked to metabolism were upregulated in these modules; Slc27a3, Fabp5 gene, fatty acid metabolism, and Ass1, L-arginine and urea metabolism, Calm1 ans S100a10, involved in calcium metabolism (Figure (8I) (Schaper and van Spriel 2018; Li et al. 2009; Lee, Kim, et al. 2020; Andersen et al. 2016; Weyd 2016; Cook et al. 2020). Antigen-cross presentation has been linked to a high expression of Serpinb9, which was clearly up-regulated in cDC1 cells in CTA1-exposed cluster cells (Figure 8I)(Mangan et al. 2017). Thus, exposure to CTA1-adjuvant dramatically changed the gene expression pattern in $\mathrm{cDC} 1$ cells leading to stimulatory functions that promoted Th17-differentiation in primed CD4 T cells as well as an improved ability to cross-present and stimulate CD8 T cell responses. 


\section{Discussion}

Previously published studies have indicated that the two lineage-specific cDC-subsets are to be viewed as functionally specialized and largely non-reduntant because the cDC1 subset promotes Th1 cell development and cross-presentation to CD8 T cells, while cDC2 cells are responsible for Th2 and Th17/Tfh cell priming (Dudziak et al. 2007; Brown et al. 2019; Durai and Murphy 2016; Anderson, Murphy, and Briseno 2018; Persson et al. 2013; Schlitzer et al. 2015; Mayer et al. 2017; Tussiwand et al. 2015; Ma et al. 2019). However, the present study challenges this notion by demonstrating that $\mathrm{cDC} 1$ cells can, under certain conditions, prime Th17 cells as we show following i.n immunizations with the CTA1-peptide-CD103 fusion protein. We provide unequivocal evidence demonstrating that the CTA1-peptide-CD103 fusion protein, indeed, acts through $\mathrm{cDC} 1$ cells and not through $\mathrm{cDC} 2 \mathrm{DP}$ cells, albeit the latter cells also express CD103. Several lines of evidence support this interpretation of our results; 1) Batf3-deficient mice, which lack $\mathrm{cDC} 1$ cells, fail to respond to the fusion protein, 2) in vitro cultures of naïve CD4 $\mathrm{T}$ cells with highly enriched cDC1 cells from i.n immunized mice respond with Th17 development and IL-17, IL-21 and IL-22 production, 3) highly enriched cDC1 cells produce IL-1 $\beta$, Il-6 and IL-23 upon stimulation with the fusion protein in vitro, and finally 4) a scRNAseq analysis of freshly sorted cDC1 and cDC2 cells revealed that cDC1 cells, rather than $\mathrm{cDC} 2$ cells, undergo extensive CTA1-enzyme dependent modifications of gene expression, some which have been linked to a Th17 priming function in earlier studies (Segura et al. 2013; Bhaumik and Basu 2017; Lee, Kim, et al. 2020; Lee, Zhang, et al. 2020; Yosef et al. 2013). This ability of CTA1-adjuvant appears to be quite unique as CpG, poly I:C and LPS, exerting their actions via Myd88/TRIF-signaling pathways and different Toll-like receptors (TLRs), promote Th1 differentiation, in agreement with previous reports (Perkins et al. 2018; Cancel et al. 2019). The CTA1-adjuvant effect on cDC1 cells was dependent on its ADPribosylating ability since an enzymatically inactive mutant did not exert adjuvant function, which we have also reported earlier (Lycke and Lebrero-Fernandez 2018). Thus, the present study has unravelled that functional plasticity can be exhibited by cDC1 cells, which were found 
to prime for Th17 cell differentiation. Using an ADP-ribosylating CD103-targeted CTA1adjuvant we achieved M2e-specific Th17 differentiation and strong protection against influenza virus infection.

In earlier work we targeted antigen to $\mathrm{CD} 103^{+} \mathrm{APCs}$ via conjugation to anti-CD103 Mab and found that both CD4 and CD8 T cells could be effectively primed (Semmrich et al. 2012). Exploiting the CD103-targeting element for delivery of a strong mucosal adjuvant, such as CTA1, was, therefore, a natural next step in this strategy. Apart from antigen-delivery we achieved a strong immunoenhancement of the APC function in $\mathrm{CD}_{103}{ }^{+} \mathrm{cDC}$ cells, resulting in augmented priming ability of both CD4 and CD8 T cells, without adding a separate adjuvant because the fusion protein itself harnessed the CTA1-adjuvant (Lycke and Lebrero-Fernandez 2018). Hence, the fusion protein provided targeting, immunoenhancement and delivery of vaccine epitopes, in one molecule. In agreement with our earlier work with anti-CD103 Mab targeting, the ScFv-approach exhibited strict dependence on CD103-expressing cDCs for its immune stimulating effects (Semmrich et al. 2012). This was evident from the lack of an effect in CD103-/- mice, and, perhaps, best noted in chimeric mice that hosted cDC1 cells, which were CD103-deficient, and failed to respond. Thus, the target population was restricted to $\mathrm{CDC} 1$ and did not depend on cDC2DP cells, despite that the latter carry CD103. In fact, huCD207DTU mice that were deficient in cCD2DP cells in mLN demonstrated full adjuvant and antigenic properties in response to the fusion protein, while Batf3-/-mice, lacking cDC1 cells, did not respond (Theisen et al. 2018; Welty et al. 2013).

To secure that we investigated migratory cDCs that had bound and taken up the fusion protein following i.n administration we employed FACS-sorting using labelled $\mathrm{Yae}^{+}$Mab that detects the E$\alpha$-peptide on the cDC cell surface (Bernasconi et al. 2020). This way we could identify the exact subset of migratory cDCs that were affected by CTA1-adjuvant and which were instrumental for priming of the CD4 T cells in the draining mLN. Noteworthy, for comparison we sorted migratory cDCs from $\mathrm{mLN}$ from unimmunized mice, cells that had been naturally activated and matured, prior to trafficking to the mLN. The scRNAseq analysis was highly 
revealing and identified a substantial modification of genes expressed in immunized as opposed to unimmunized $\mathrm{cDC} 1$ cells, while $\mathrm{cDC} 2$ cells were less affected and found within the same cluster in the UMAP. The cDC1 cells from unimmunized mice distributed mainly to clusters 1 and 2, while a majority of CD103-targeted cells were found in clusters 3 and 4 . The greater impact on cDC1 cells agreed well with the absolute requirement for cDC1 cells, lost in Batf3/-, for the adjuvant effect (Theisen et al. 2018). Thus, cDC1 cells were distributed in 4 different clusters and a pseudotime trajectory analysis identified significant gene expression changes in CTA1-exposed versus adjuvant-unexposed cDC1 cells. These changes affected genes associated with cell migration, cell signalling and co-stimulation. More specifically, we observed increased expression of genes associated with Th17-priming (IL-1b, IL-6, Tgfbl, Tgfb2, Tnfsf4 (Ox40L), Tnfrsf9 (CD137) IL-12b (p40), Timd4, Cd80, Cd86 ) along the trajectory in cDC1 cells exposed to CTA1-adjuvant (Segura et al. 2013; Anderson et al. 2020). Strikingly, a GSEA analysis based on gene lists from a previous publication of human inflammatory DC and a Th17-inducing function, supported our interpretation that the cDC1 cells had acquired a Th17 promoting gene signature following CTA1-exposure (Segura et al. 2013). Noteworthy, the gene list used represented inflammatory DC that were thought to be derived from monocytes, genetically more like murine $\mathrm{CD} 11 \mathrm{~b}^{+} \mathrm{cDC} 2$ cells and not $\mathrm{cDC} 1$ cells (Dutertre et al. 2019; Segura et al. 2013). But, despite this our comparative analysis showed significant gene enrichment for a Th17-inducing function, similar to the "pro-Th17 gene signature" reported earlier for inflammatory DCs in humans, i.e CD163+DC3s , which are related to cDC2s (Dutertre et al. 2019; Segura et al. 2013). Furthermore, the up-regulated expression of $I L-1 b, I L-6, T g f b 1$ and $I L-12 b$ (p40) genes we found are clearly linked to a Th17 promoting function by cDCs (Dutertre et al. 2019; Segura et al. 2013). Apart from the upregulated expression of these known Th17-promoting genes we identified several DEG typifying activated cDCs (Hirota et al. 2012; Segura et al. 2013; Durai and Murphy 2016). For example, up-regulated expression of Stat3, Stat4 , Cytip, Scin, Ccr7, Anxa2, Cd86 and Cd81 genes are all hallmarks of highly activated DCs and could greatly contribute to an enhanced T cell priming efficacy by facilitating cDC1 cell migration and/or antigen presentation in the 
immune synapse (Zuidscherwoude et al. 2017; Mittelbrunn et al. 2002; Watford et al. 2006; Schaper and van Spriel 2018; Theodoridis et al. 2011; Weyd 2016). Serpinb9 expression is an intracellular marker for antigen-cross presentation by cDC1 cells (Mangan et al. 2017). The interaction between tetraspanin CD81 and MHCII molecules is well documented in APCs, although CD81-/- mice have not been investigated for poor Th17 development, only impaired Th2-cell immunity and antibody production, have been reported (Schaper and van Spriel 2018; Zuidscherwoude et al. 2017). Moreover, among the DEGs with strongest association to Th17development, we find Fabp5, Rel and Ox4OL (Carmody et al. 2007; Li et al. 2009). Expression of Rel links cDC1 cells to the transcription factor NF- $k \mathrm{~B}$ and signaling events that control $I L$ 23 p19 gene expression and in this way influences Th17 differentiation in CD4 T cells (Carmody et al. 2007). The involvement of fatty acid metabolism in Th17 differentiation has been documented before and appears to link DC activity to Stat3-signaling and Th17differentiation in CD4 $\mathrm{T}$ cells ( $\mathrm{Li}$ et al. 2009; Lee, Kim, et al. 2020). Tnfsf4-expression, encoding OX40L, has been associated with autoimmunity and Th17 cell differentiation, but reported to be expressed only in cDC2 cells and not in cDC1 cells (Webb, Hirschfield, and Lane 2016; Zhang et al. 2010).

We have previously demonstrated that the lung resident M2e-specific Th17 memory cells convey strong protection against influenza virus infection following i.n immunization with CTA1-M2e-DD (Eliasson et al. 2018). In the present study we improved immunogenicity of the fusion protein by changing the targeting element from DD to scFvCD103 and achieved significantly stronger protection, roughly 35-100 fold more effective, compared to the DDconstructs. This way the efficiency in targeting the fusion protein to cDC1 cells was much improved, although we learned that both the -DD and -CD103 constructs relied on the cDC1 subset for their adjuvant functions. This latter observation was unexpected as cDC2DP cells were also found to bind the fusion proteins, but these cells failed to replace cDC1 cells in Batf3/- mice. In particular, the -DD constructs, which bound cDC1 and cDC2 cells equally well, were found poorly effective in Batf3-/- mice. Similar to intact CT-adjuvant, both CTA1-fusion proteins were effective at augmenting CD8 T cell priming by cross-presenting cDC1 cells 
(Olvera-Gomez et al. 2012). This ability has recently been successfully explored by us in a therapeutic vaccine model against tumor metastasis where we observed augmented tumorspecific CTL-induction, which agrees well with previous studies on CD103-targeted vaccine approaches and CT-adjuvant (M. Arabpour et al, unpublished data)(Hartung et al. 2015; Apte et al. 2013).

Functional plasticity in $\mathrm{cDCs}$ has previously been observed within the $\mathrm{cDC} 2$ lineage subsets and was reported to depend on the level of expression of the Irf4 and Klf4 genes ((Lee, Zhang, et al. 2020)). While increases in intracellular cAMP repressed these genes and modified the function of cDC2 cells to prime Th17 rather than Th2 responses, there were no modifying effects of cAMP-increases reported in splenic cDC1 cells (Lee, Zhang, et al. 2020)7. Whereas we found no dependeny on cDC2 cells for the adjuvant effect of the targeted CTA1-adjuvant our results differ from those of Lee et al in that they had no effect of cAMP-enhancers, such as CT, in cDC1 cells (Lee, Zhang, et al. 2020). However, while CTA1 is the cAMP-inducing element in CT holotoxin it is probable that our CTA1-fusion protein does not induce cAMP. Indeed, preliminary studies have indicated that the effect of the fusion protein may be independent of Gs $\alpha$-expression and cAMP-induction in CD11c cells (Lycke and LebreroFernandez 2018). Additional experiments are required, though, to prove this point. Importantly, the CD103-targeting ScFv-element in itself had no adjuvant effect and could not promote Th17 cell development in vitro. This effect was rather a consequence of CTA1-actions on the cDC1 cells, which included enhanced co-stimulation and cytokine production with increased IL-1 $\beta$, IL-6 and IL-23 levels in cultured cDC1 cells after exposure to the adjuvant. This was in contrast to the TLR-binding adjuvants, $\mathrm{CpG}$, poly I:C and LPS, which all stimulated Th1 and IFN $\gamma$ production and they had no promoting effect on ror $\mathrm{t}^{+}$Th17- or IL-17 responses in cultured cDC1 cells (Perkins et al. 2018; Cancel et al. 2019). To our knowledge the present study represents a major shift of paradigm as it demonstrates that $\mathrm{cDC} 1$ cells show plasticity and under certain conditions, can be inducers of Th17 responses, a function previously thought to 
be restricted to $\mathrm{cDC} 2$ cells. This observation not only challenges the idea of a functionally nonredundent lineage-restricted $\mathrm{cDC} 1$-subset, but it is likely to have fundamental impact on future vaccine adjuvant development. 


\section{Acknowledgements}

The study received funding from the People Programme (Marie Curie Actions) of the European Union's Seventh Framework Programme FP7/2007-2013/ under REA grant agreement number 607690. It was also supported in parts by research funds from the Knut and Alice Wallenberg Foundation KAW 2013.0030, the Swedish Foundation for Strategic Research SB12-0088, The Swedish Cancer Foundation, The Swedish Research Council, the EU project UNISEC, LUA/ALF ALFGBG-531021 and the Lundberg foundation. A grant from SciLife Sweden for bioinformatics analysis of the scRNAseq data was graftefully received. The authors would like to thank Jan-Olof Andersson and Richard Christison at MIVAC Development AB for production of the fusion proteins. Special thanks to the NIH Tetramer Core Facility for producing the unique MHC class II restricted M2e-tetramer. We thank D. Kaplan for generously letting us use the huCD207DTA mice and the staff at the Laboratory for Experimental Biomedicine at the University of Gothenburg for breeding the indicated mouse strains.

\section{Author contributions}

MA and NL designed the study and planned the experiments. MA, CL and KS performed the experiments and analyzed the data. VB and DA performed bioinformatics and modeling analysis. MA, CL, KL, DA, WA and NL analyzed and interpreted the data. MA and AS made the figures. MA, DA and NL wrote the manuscript.

\section{Competing interests}

The authors declare no competing interests 


\section{Materials and Methods}

\section{Mouse strains and immunizations}

Female mice, 6-10 weeks of age, were used for all experiments. C57B1/6 (CD45.2 $\left.{ }^{+}\right)$and Balb/c wild-type (WT) mice were purchased from Janvier Labs (Paris, France). OT-II (CD45.1 ${ }^{+}$), OTI (CD45.1 ${ }^{+}$), E $\alpha$-TCR Tg mice (Viret, He, and Janeway 2000) and Batf 3-/- mice were bred at EBM animal facility at the University of Gothenburg. HuCD207/DTA (Welty et al. 2013) and Itgea (CD103-/-) knock out mice on the C57B1/ 6 background ( were bred at Lund University (Lund, Sweden) and transported to Gothenburg for experiments. Animal experiments were approved by the local Ethics Committee

C57Bl/6 or Balb/c wild-type (WT) mice were immunized i.n with a single dose or 3 doses with 10 days apart, as indicated. Balb/c mice were used only for influenza virus protection studies because the M2e-peptide is restricted to $\mathrm{H}-2^{\mathrm{d}}$ (Eliasson et al. 2018). For comparative studies of adjuvant efficacy between CD103- and DD-constructs immunizations were also performed i.p, as indicated, with admixed soluble antigens; $5 \mu \mathrm{g}$ 4-Hydroxy-3-nitrophenylacetyl-Chicken Gamma Globulin( NP-CGG or tetanus toxoid (TT) (Statens Serum Institute, Copenhagen, Denmark) Fusion proteins and ovalbumin were given in $5 \mu \mathrm{M}$ doses, unless stated otherwise. Serum and bronchoalveolar lavage (BAL) was sampled 6-8 days after the last immunization or 18 days after inoculation of virus and stored frozen at $-20 \mathrm{C}$ until analyzed for specific antibody titers by ELISA.

\section{Influenza virus challenge infection}

Immunized or PBS control Balb/c mice were subjected to a virus challenge infection. Briefly, 2-3 weeks after immunizations groups of 10 mice were inoculated with an i.n dose of PR8 A/Puerto Rico/8/34 (H1N1) virus at $4 \times$ LD50, (corresponding to $2.5 \times 10^{3}$ TCID50). Morbidity (body weight) and mortality were monitored daily for 2 weeks (Eliasson et al. 2018). Mice were sacrificed when reaching a weight loss $>30 \%$.

\section{Fusion proteins}


CTA1-peptide-CD 103 fusion proteins were produced; Briefly, anti-CD103 single chain antibody (CD103 scFv) cloning and construction was done using regions from both variable heavy (VH) and light (VL) immunoglobulin chains from the anti-CD103 antibody produced by the M290 hybridoma. The desired regions from M290 VH and VL chains were RT-PCR amplified and sequenced. Amplified regions were linked together via the genetic sequence corresponding to the 4 GGGGS linker region to form scFv CD103. This was followed by linking a gene sequence corresponding to the cholera toxin A1 enzyme (CTA1) subunit or its mutated and enzymatically inactive CTA1(R9K) gene sequence which was fused N-terminally of the anti-CD103 scFv fragment. Constructs with peptides incorporated into the fusion proteins were designed as follows; fusion proteins that carried genetic sequences encoding the MHC class II H-2 ${ }^{\mathrm{b}}$ restricted E $\alpha$ peptide 52-68 (ASFEAQGALANIAVDKA ), the OVA p323 peptide (ISQAVHAAHAEINEAGR), the MHC class I restricted OVA SIINFEKL peptide and the MHC class II H-2d-restricted influenza virus specific M2e peptide (ETPIRNEWGSR) were engineered. Corresponding fusion proteins with -the DD-dimer instead of the scFvCD103 were expressed and purified as described before (Agren, Norin, et al. 1999). The purified material contained no endotoxin (0.1EU per mg of protein), it bound avidly to IgG in solid phase and CTA1 was enzymatically active as determined with the agmatine assay as described (Agren, Norin, et al. 1999).

\section{Adoptive cell transfers and chimeric mice}

Splenic OT I or OT II T cells from CD $45.1^{+}$donor mice were labeled with Carboxyfluorescein succinimidyl ester (CFSE) (Life Technologies) and 1 x $10^{6} \mathrm{~T}$ lymphocytes were adoptively transferred into C57B1/6 (CD45.2+) WT recipient mice . Immunizations were given on day 1 after transfer of cells. Bone marrow (BM) chimeras were made by i.v. transfer of $2 \times 10^{6} \mathrm{BM}$ donor cells from Batf3 and CD103-/- or WT mice into irradiated (1000 rad) recipient WT mice. BM reconstitution was achieved by 50/50\% BM from Batf3-/- and CD103-/- or WT mice, respectively, so that chimeric mice would have cDC1 cells without (CD103-/-) or with CD103 
(WT) expressing cells, while both strains would have cDC2 cells with CD103. Experiments with chimeric mice were initiated at 6 weeks after BM reconstitution.

\section{In vitro cell cultures}

cDC-T cell co-cultures were established using highly enriched (>93\%) cDCs from mLN taken from immunized or unimmunized WT mice. Briefly, highly enriched cDC1 cells were obtained by stepwise purification using MACS-and negative selection with pan DCs selection (Miltenyi biotec) followed by positive selection with biotin tagged anti-XCR1 beads for maximum cDC1enrichment (CD103+ CD11b- DCs) (Stem cell technologies). Purified cDC1s ( $\left.2 \times 10^{4}\right)$ were then co-cultured (1:10) with autologous, naive OT-II or E $\alpha$-TCR Tg CD4 T cells $\left(2 \times 10^{5}\right)$ in 96 well U-bottom plates in IMDM medium containing with 10\% FCS (Sigma Aldrich), $1 \mathrm{mM}$ sodium pyruvate (Gibco), $1 \mathrm{mM}$ beta mercaptoethanol (Sigma) and $50 \mu \mathrm{g} / \mathrm{ml}$ Gentamycin (Gibco) and plated ( $2 \times 10^{5}$ cells/well) presence or absence of ovalbumin $(5 \mu \mathrm{g} / \mathrm{ml})$ or E $\alpha$-DD $(1 \mu \mathrm{M})$ antigen and adjuvants; CTA1-II-CD103 $(1 \mu \mathrm{M})$, Poly I:C $(10 \mu \mathrm{g} / \mathrm{ml})$, LPS $(10 \mu \mathrm{g} / \mathrm{ml})$ and $\mathrm{CpG}(2.5 \mu \mathrm{g} / \mathrm{ml})$ for $48 \mathrm{~h}$ at $5 \% \mathrm{CO}_{2}$ and $37^{\circ} \mathrm{C}$. T cell proliferation was determined by flow cytometry using the CFSE-dilution (Thermo Fisher Scientific) assay. Cytokine concentrations were determined by ELISA and intracellular staining for IFN $\gamma$ used labeled anti-IFN $\gamma$ Mab ( BD Biosciences) and flow cytometry.

Lymphocytes were isolated from the mLN or lung of immunized or immunized and challenged mice. Briefly, cells from the mLN were isolated by mechanical disruption and lung tissues were enzymatically digested using a kit from Miltenyi Biotec (Bergisch Gladbach, Germany) to obtain single-cell suspensions. Triplicate cultures were performed in 96-well plates (Nunc) and $\mathrm{T}$ cell recall responses were allowed 3 days in the presence of medium only or different concentrations of antigen, as indicated in the Figure legends. CD4 T-cell proliferation in vitro used CFSE-labeled OT-I or OT-II cells and cytokine determinations were performed on supernatants. 


\section{Antibody and cytokine determinations}

The ELISPOT assay was used to determine IL-17 or IFN $\gamma$ producing cells from isolated mLN or lung lymphocytes. The dual kit for IL-17/IFN $\gamma$ or single IFN $\gamma$ spot forming cell (SFC) assessments was used according to the manufacturer's instructions (Immuno Spot). Briefly, single cell suspensions of mononuclear cells were seeded in 96-well plates at $1 \times 10^{5}$ cells/well and $1 \mu \mathrm{M}$ of peptide was added and the plates were incubated for 24 houres $\left(5 \% \mathrm{CO}_{2}, 37^{\circ} \mathrm{C}\right)$. Cytokine ELISPOTs were evaluated using a CTL ImmunoSpot analyzer. Cytokine determinations using ELISA were used for assessments of cytokines in culture supernatants. Prior to analysis samples were stored at $-80 \mathrm{C}$ until further analyzed. Cytokine concentrations were determined in supernatants by ELISA (Duoset, R\&D Systems, UK) and the concentrations of IFN $\gamma$, IL22, IL21, IL23, IL6, IL1 $\beta$ and IL-17A was done and given in pg/ml using a standard curve provided by the manufacturer.

\section{Flow cytometry}

Flow cytometry studies were performed as described in detail elsewhere. Briefly, dead cells were identified by 7-amino-actinomycin D or Live/Dead Aqua LIVE/DEAD Fixable Dead Cell Staining Kit (Life Technologies). Intracellular FACS stainings were achieved by the Fixation/Permeabilization Kit (eBioscience) according to the manufacturer's instructions. Flow cytometry used a FACSAriaII or LSRII (BD Biosciences) and cells were analyzed using FlowJo X (Tree Star). Cell sorting of migratory cDC was done using a FACSAriaII (BD). The following fluorophores and conjugated antibodies were used for phenotypic analysis; M2especific tetramer-PE and control CLIP-PE and staining was performed at 37C for $30 \mathrm{~min}$ and followed by labeling on ice with cell surface specific markers for $30 \mathrm{~min}$ as follows; CD4 Alexa-700, CD8 AF647, CD3e BUV737, CD45.1 APCe Flour 780, CD69 PE-Cy7 and CD44 FITC. For labeling cDCs, we used CD11c BV421, MHC II (IA-E) Alexa-700, CD11b FITC, CD103 PE, and XCR1 . APC AF647, CD19- APC-cy7 and F4/80 BV711 were used for a dump channel. Intracellular staining for detection of transcription factors; Tbet BV421, ROR $\gamma \mathrm{t}$ PE- 
CF594, GATA3 PE . T cell proliferation was assessed by CFSE-dilution which applied to both in vivo isolated cells as well as cells from in vitro cultures.

\section{In vivo cytotoxicity assay}

Following transfer of $1 \times 10^{5}$ OT I cells i.v. to autologous WT C57B1/6 mice these were immunized i.n with $5 \mu \mathrm{M}$ of the fusion proteins $12 \mathrm{~h}$ later. Splenocytes were taken from naïve mice and pulsed with SIINFEKL peptide $(1 \mu \mathrm{g} / \mathrm{mL}$ peptide $)$. Incubations were done at $4^{\circ} \mathrm{C}$ for 1.5 hours followed by $37^{\circ} \mathrm{C}$ incubation for 30 minutes. The cells were labeled with CFSE $(2 \mu \mathrm{M})$ (target cells) or unlabeled Far Red $(2 \mu \mathrm{M})$ (bystander cells) without adding peptide (negative control); $10 \times 10^{6} \mathrm{CFSE}$ and $10 \times 10^{6}$ Far Red labeled splenocytes $\left(20 \times 10^{6}\right.$ cell transfer /mouse ) were mixed and transferred to autologous WT recipient mice. After $20 \mathrm{~h}, \mathrm{mLN}$ and spleens of immunized mice were stained with Live dead aqua dye and analyzed by flow cytometry. Cytotoxicity was then determined as follow:\% killing $=\left(1-\left(\frac{\% \text { CFSE }}{\% \text { Far Red }}\right)\right) \times 100$.

\section{Single cell RNAseq and Seurat and Monocle 3 analysis}

Migratory cDCs (MHC II high CDllc high) from naïve/PBS-treated or i.n fusion protein immunized mice were isolated from mLN from a pool of 7-8 C57Bl/6 mice and FACS -sorted on a Aria II cell sorter (BD Bioscience). cDCs exposed to the fusion proteins were identified on the basis of their expression of the labeled E $\alpha$-peptide+ MHC classII -specific Yae Mab. Labeling of cells prior to sorting by flowcytometry (Fusion, BD) included the following specific Mabs Yae, anti-CD4, anti-CD8, anti-B220 and anti-F4/80, as described (Bernasconi et al. 2020). Cells were sorted into IMDM, before being pelleted and resuspended in IMDM-10\% FBS. Approximately 5000 sorted celles were loaded on the 10x Chromium Controller (10x Genomics). The scRNA-seq libraries were prepared following the user guide manual provided by the 10X Genomics company. Data from scRNA-seq of DC sorted samples were individually processed using cell ranger analysis pipeline (10X genomics platform) and reads aligned on the mm10 genome assembly. The output form Cell Ranger then further analysed with Seurat Seurat 
3.0. Raw UMI count matrices were loaded and merged into a single Seurat object. Cells were discarded if they met any of the following; percentage of mitochondrial counts greater than 6 percent per cell, number of unique features either below 200 or above 3500, T cell genes ('Cd3d', 'Cd3e', 'Cd3g', 'Trac', 'Trbc1', 'Trbc2') greater than 0.1 percent and B cell genes ('Cd79a', 'Cd79b', 'Ms $4 a 1^{\prime}$, ' $C d 19$ '), greater than 0.1 percent. Gene counts were log normalized and mean centered and scaled by their standard deviation and the following variables regressed out : the number of UMI and the percentage of mitochondrial counts. Finally, cells were clustered and UMAP applied to further reduce dimensionality for visualization. Differential gene expression was performed using the FindAllMarker function with Wilcoxon Rank Sum test and minimum of 0.25 log fold-change. For analysis using Monocle v3, the Seurat object was imported using the as.cell_data_set function from SeuratWrappers and further processed. Pseudotime analysis was performed using standard settings and with root node selection in cluster 0 . Modules of co-regulated genes, differentially expressed across pseudotime, were identified using the function find_gene_modules using a list of Louvain resolution and selecting the one with highest value.

\section{Gene set enrichment analysis (GSEA)}

For GSEA analysis, differentially expressed genes for CTA1 (DD- and CD103) versus PBS in cDC1 population were calculated using the Wilcoxon rank sum test via the wilcoxauc function of the presto package using default parameters (including Benjamini-Hochberg false discovery rate correction) and filtered on $\log F C>1$ and padj $<0.05$. GSEA was run on pre-ranked genes using the fgsea package (Korotkevich, G., V. Sukhov, and A. Sergushichev. 2019. Fast gene set enrichment analysis. bioRxiv 060012). For each enrichment graph we report p, padj (FDR q) and NES (enrichment score normalized to mean enrichment of random samples of the same size) values in the figure. 


\section{Figure Legends}

\section{Figure 1}

\section{The scFv CD103 fusion protein specifically binds $\mathrm{CD103}^{+}$DCs populations}

Schematic representation and in silico model of the adjuvant active fusion proteins; CTA1-IDD, CTA1-I/II-DD, CTA1-I-CD103 and CTA1-I/II-CD103 molecules, carrying the MHC Irestricted SIINFEKL-peptide or class II-restricted p323-peptide from ovalbumin (A). Western blot analysis of the CTA1-I-DD (1), CTA1-I/II-DD (2), CTA1-I-CD103 (3) and CTA1-I/IICD103 (4) molecules (B). The ADP-ribosylating activity of CT holotoxin and the CTA1enzyme or mutant CTA1R7K in the respective fusion proteins as assessed by the agmatine in vitro test. Means (C). Binding-specificity of the fusion proteins was assessed in vitro by gating on migratory DCs (MHC II ${ }^{\text {high }}$ CD11 $c^{\text {high }}$ ) from mesenteric LN (MLN) from WT or CD103-/C57Bl/6 mice and flow cytometry with labeled DC-relevant Mabs (CD11c, MHC-II, CD103, CD11b) and labeled anti-Flag Mab to detect the fusion proteins used at $1 \mu \mathrm{M}$ conc (D). Similar to $\mathrm{D}$, but this in vitro analysis used WT migratory DCs or B cells $\left(\mathrm{CD} 19^{+}\right)$from mediastinal LN (mLN) to assess the ability of the fusion proteins (MFI of labelled anti-Flag Mabs) at 1 and $0.1 \mu \mathrm{M}$ concentrations to bind the different DC-subsets; CD103 ${ }^{+} \mathrm{CD} 11 \mathrm{~b}-\mathrm{CD}^{-} 03^{+} \mathrm{CD}^{-11 b^{+}}$, $\mathrm{CD}^{103} \mathrm{CD}^{-} 1 \mathrm{~b}^{+}$or $\mathrm{B}$ cells using flow cytometry (E). Results are representative of three independent experiments with mean values $\pm \mathrm{SD}$ for means of triplicate cultures $(\mathrm{D}, \mathrm{E})$. Statistical significance was calculated using student t-test with $\mathrm{p}$-value $* \mathrm{p}<0.05, * * \mathrm{p}<0.01, * * *$ $\mathrm{p}<0.001$.

\section{Figure 2}

The CD103-targeted CTA1-adjuvant acts through cross-presenting cDC1 cells effectively priming CD8 T cells and CTLs

C57B1/6 mice were i.n. administered with $5 \mu \mathrm{M}$ of OVA, CTA1-I/II-DD or CTA1-I/II-CD103 and 20h later the frequency of migrating $\mathrm{CD} 103^{+} \mathrm{CD} 11 \mathrm{~b}^{-} \mathrm{cDC} 1$ cells expressing SIINFEKL- 
peptide + MHC I complexes in the mLN was assessed using flow cytometry and a labeled complex-specific Mab and an isotype control Mab. MFI values are given as means \pm SD of three independent experiments (A). An adoptive transfer model with carboxyfluorescein succinimidyl ester CFSE-labeled OT-I TCR Tg donor CD8 T cells (CD45.1 $\left.{ }^{+}\right)$injected into WT C57B1/6 (CD45.2 $2^{+}$mice followed by a single i.n priming immunization with an equimolar dose (SIINFEKL peptide) of ovalbumin (OVA), CTA1-I/II-DD or CTA1-I/II-CD103. At 4 days post immunization CD45.1 ${ }^{+}$OT I CD8 T cell proliferation was assessed in freshly isolated mLN cells by flow cytometry. The CFSE-dilution profiles were determined and values are given as the mean frequency $\pm \mathrm{SD}$ of proliferating OT I cells of all CD8 $\mathrm{T}$ cells, proliferation index and frequency of responding OT I CD8 T cells, as indicated (see M \& M section) Representative dot-blots of three independent experiments giving similar results and values are given as means \pm SD of 3-5 mice in each group (B). Determination of SIINFEKL-specific IFNyELISPOTs in OT I CD8 $\mathrm{T}$ cells stimulated with recall ovalbumin at 5 days following i.n immunizations with the fusion proteins. Values are given as means \pm SD of 5 mice in each group and one representative experiment of three is shown (C). Induction of CTL responses in C57BL6 mice following i.n. immunization with a 5 $\mu \mathrm{M}$ dose of OVA, CTA1-I/II-DD, CTA1I/II-DD or CTA1- II-CD103 (control w/o SIINFEKL peptide) fusion protein. After 1 week mice were injected i.v. with CFSE-labelled splenocytes pulsed with 1uM SIINFEKL peptide (Target cells) or unpulsed control splenocytes (Bystander cells). After 20h freshly isolated mLN (mediastinal lymph node) cells were analyzed for specific lysis of peptide expressing target cells by flow cytometry. Values are means \pm SD of 2 independent experiments giving similar results with 2-3 mice per group (D). Statistical significance was calculated using ANOVA with Dunnett's post-test $(\mathrm{A}, \mathrm{B}, \mathrm{C})$ or Student's $t$-test (D). ns; not significant; $\mathrm{p}$-values * $\mathrm{p}<0.05$, ** $\mathrm{p}<0.01, * * * \mathrm{p}<0.001, * * * * \mathrm{p}<0.0001$

\section{Figure 3}


Balb/c mice were i.n immunized 3 times with $5 \mu \mathrm{M}$ of CTA1-3M2e-CD103 (n=10) or CTA13M2e-DD $(n=10)$ and immune protection was determined 3 weeks after the final immunization by a live challenge infection with $4 \times \mathrm{LD}_{50}$ of a highly virulent mouse adapted PR 8 strain (Eliasson et al. 2018). The frequency of surviving animals and changes in body weight was monitored over time. Anti-M2e IgG2a (most protective antibodies) in serum and IgA in BAL after the challenge infection in one representative experiment of three giving similar results was assessed by ELISA and given as $\log _{10}$ titers \pm SD (A). Representative FACS plots of M2etetramer-specific lung CD4 $\mathrm{T}$ cells isolated from i.n immunized or naïve control mice, as indicated. Prior to the challenge infection the frequency of M2e-specific CD4 T cells that were $\mathrm{CD} 9^{+}$or $\mathrm{CD}^{-} 9^{-}$and the representation of Th17 cells (roryt $\mathrm{t}^{+}$) is given as $\% \pm \mathrm{SD}$ of all M2especific CD4 T cells $(n=3)$. Results are from three independent experiments giving similar results (B). Statistical significance was calculated using ANOVA with Dunnett's T3 post-test analysis: ns; not significant, $\mathrm{p}$-values $* * \mathrm{p}<0.01, * * * \mathrm{p}<0.001$ and $* * * * \mathrm{p}<0.0001$

\section{Figure 4}

\section{The CD103-targeted adjuvant effectively primes Th17 cells following i.n immunizations}

Adoptive transfer i.v of CFSE-labeled OT II TCR Tg CD4 T cells (CD45.1+ ) to WT C57Bl/6 mice $\left(\mathrm{CD} 45.2^{+}\right)$was followed by a single i.n immunization with $5 \mu \mathrm{M}$ OVA, CTA1-II-DD or CTA1-II-CD103 the next day (equimolar doses of p323). On day 5 mice $(n=5)$ were analyzed for OT II cell proliferation in the mLN (mediastinal lymph node) by assessing the dilution of CFSE-label by flow cytometry. The enzymatically inactive CTA1R9K-II-CD103 fusion protein was evaluated for its adjuvant activity. The frequency of expanding OTII cells of all CD4 T cells is given as mean $\% \pm \mathrm{SD}$ of 3-5 mice in each group and pooled data from 3 independent experiments is shown. (A). The ability to prime OT II CD4 T cells in the draining $\mathrm{mLN}$ at different days post-immunization was assessed as in A. OTII cells were injected i.v. on days 1,4 or 8 after i.n immunizations with the fusion proteins and values are given as means \pm SD of 5 mice in each group $(n=2)(B)$. The differentiation into Th17 or Th1 OT II cells in the mLN 


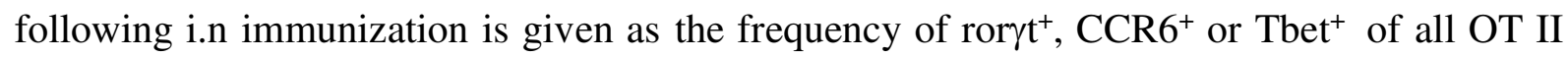
CD4 T cells. Values in each category are given as mean $\% \pm \mathrm{SD}$ of all OT II cells, as indicated, and these are representative of three independent experiments with 2-6 mice in each group (C). IL-17A and IFN $\gamma$-specific ELISPOT analysis of OT II CD4 T cell recall responses to p323 peptide was performed in triplicates by freshly isolated $\mathrm{mLN}$ cells following a single i.n immunization of the respective fusion protein. Values are given as mean $\pm \mathrm{SD} S F C / 10^{5}$ cells of three independent experiments with 3-5 mice per group (D). Statistical significance was calculated using ANOVA with Dunnett's posttest (A, B) or Student's $t$-test (C). p-values are given as; $* \mathrm{p}<0.05, * * \mathrm{p}<0.01, * * * \mathrm{p}<0.001, * * * * \mathrm{p}<0.0001$. Finally, OT II cells from i.n immunized mice were subjected to a total RNAseq analysis using pooled samples from mLN from 3 mice. The heat map shows relative expression of Th17-relevant genes (rorc, rora, $I L$ 17a, IL-22, Ccr6) in the different groups; OVA, CTA1-I/II-CD103 and CTA1-I/II-DD. This is a representative analysis of two independent experiments giving similar results (E).

\section{Figure 5}

\section{The CTA1-II-CD103 adjuvant effect is mediated via cDC1 cells}

Mice with different restrictions in their DC-subset repertoire were immunized i.n following adoptive transfer of CFSE-labelled OT II cells (CD45.1+ ) according to the protocol used for Fig.4. WT C57BL/6, transgenic CD103-/-, BATF3-/- or huCD207/DTA mice and littermate controls were immunized with $5 \mu \mathrm{M}$ CTA1-II-DD (blue) or CTA1-II-CD103 (red) and migratory DCs $\left(\mathrm{MHC}^{\text {high }}, \mathrm{CD} 11 \mathrm{c}^{\text {high }}\right)$ and OT II cells were isolated and analyzed by flow cytometry after 20h (cDCs) and 4 days (OT II cells), respectively. DC-subsets were identified using Mabs specific for CD103, CD11b and XCR1, as indicated. Representative dot-blots of DCs in mLN (mediastinal lymph node) following i.n immunizations are given for each mouse strain (A). OT II cell proliferation was determined following a single i.n immunization and values are given as mean $\% \pm \mathrm{SD}$ of OT II cells of all isolated CD4 $\mathrm{T}$ cells in the respective group ( $n=3-5)$ and representative of three independent experiments giving similar results (B). 
Bone marrow chimeric $\mathrm{cDC} 1\left(\mathrm{CD}_{103^{-/}}\right)$and $\mathrm{cDC} 2\left(\mathrm{CD} 03^{+/+}\right)$mice were generated by reconstituting irradiated littermate C57B1/6 mice with 50/50\% BM from Batf3-/- and CD103/- or WT mice (7,5 million BM cells /per mouse). The distribution of XCR $1^{+}$cells among cDC1 cells from reconstitutions with CD103-/- or WT BM $(n=4)$ is shown in the dot-blot. Following adoptive transfer of OT II cells $\left(\mathrm{CD} 45.1^{+}\right)$into the chimeric mice and i.n immunizations as in A we show mean $\% \pm \mathrm{SD}$ of responding OT II cells of all isolated CD4 T cells in mLN of the respective group ( $n=3-5)$ in two independent experiments $(C)$. Adoptive transfer of OT I cells into mice with different cDC-deficiencies as in A, followed by i.n immunization with the fusion proteins and assessments of the efficiency of cross-presentation and priming of MHC class I restricted CD8 T cell responses. OT I cell $\left(\mathrm{CD} 45.1^{+}\right)$responses were determined and values are given as mean $\% \pm \mathrm{SD}$ of all isolated CD8 $\mathrm{T}$ cells in the respective group $(\mathrm{n}=3-5)$; pooled data from three independent experiments giving similar results (D). Statistical significance was calculated using ANOVA with Dunnett's posttest and p-values are given as; * $\mathrm{p}<0.05$, *** $\mathrm{p}<0.001, * * * * \mathrm{p}<0.0001$

\section{Figure 6}

\section{CTA1-exposed cDC1 cells are potent inducers of Th17 differentiation}

Single cell suspensions of immunized or unimmunized $\mathrm{mLN}$ (mediastinal lymph node) mononuclear cells were highly enriched (>90\%) for cDC1 $\left(\mathrm{CD}_{103}{ }^{+}, \mathrm{CD} 11 \mathrm{~b}^{-}\right)$cells by positive selection using biotinylated XCR1 Mabs at 20h following an i.n dose of 5 $\mathrm{M}$ CTA1-II-CD103. The enrichment strategy is shown with untreated cDCs from unimmunized mice and sorted cDC1 cells from mLN of immunized mice (A). Enriched migratory cDC1 cells (MHChigh, $\mathrm{CD} 11 \mathrm{c}^{\text {high }} \mathrm{XCR}^{+}$) from unimmunized or immunized mice were incubated in triplicates for 3 days with freshly isolated OT II T cells (1:10) labeled with CFSE and the histogram shows proliferating OT II cells in cultures with $\mathrm{cDC} 1$ from immunized or unimmunized mice and values are given as mean $\% \pm \mathrm{SD}$ of pooled $\mathrm{cDC} 1$ cells from 3-5 mice in each group and two independent experiments (B). The frequencies are given as mean $\% \pm \mathrm{SD}$ of Th17 $\left(\mathrm{ror}_{\mathrm{t}} \mathrm{t}^{+}\right)$and Th1 $\left(\right.$ Tbet $^{+}$) CD4 $\mathrm{T}$ cells on day 3 in these cultures of proliferating OT II cells with 
unimmunized as opposed to immunized cDC1 cells (C). Cytokine production, as indicated, in these cultures with unimmunized or immunized cDC1 cells is given in $\mathrm{pg} / \mathrm{ml} \pm \mathrm{SD}$ for each group (D). In vitro triplicates cultures with enriched cDC1 cells from unimmunized mice were

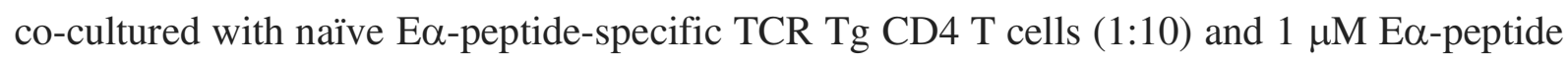
together with or without different adjuvants added simultaneously; CTA1-II-CD103 $(1 \mu \mathrm{M})$, Poly I:C $(10 \mu \mathrm{g} / \mathrm{ml})$, LPS $(10 \mu \mathrm{g} / \mathrm{ml}), \mathrm{CpG}(2,5 \mu \mathrm{g} / \mathrm{ml})$ or unstimulated $(\backslash)$, as indicated. The frequency of Roryt ${ }^{+}$or Tbet $^{+}$E $\alpha$-specific TCR Tg CD4 T cells in cultures was determined on day 3 and the frequency of intracellular IFN $\gamma^{+}$cells was assessed by flow cytometry using labeled specific antibodies. Two independent experiments (I and II) are shown giving similar results (E). Statistical significance was calculated using ANOVA with Dunnett's posttest and $\mathrm{p}$-values are given as; $* * * * \mathrm{p}<0.0001$

\section{Figure 7}

Single-cell RNAseq analysis of migratory DCs in mLN following i.n immunizations with a CD103-targeted adjuvant

Migratory cDC-subsets that had been exposed to a single dose i.n of $50 \mu \mathrm{M}$ CD103 or -DD constructs with incorporated E$\alpha$-peptide were identified by labeled Yea-Mab that binds E $\alpha$-peptide + MHC class II (I-A $A^{b}$ (Bernasconi et al. 2016). This strategy secured that only cDCs exposed to CTA1-adjuvant in vivo were included in the analysis and these were compared to $\mathrm{cDCs}$ from unimmunized mice. Migratory cDCs were sorted by FACS and subjected to a scRNAseq analysis using the 10X Chromium platform. Cluster analysis was performed using Seurat and the UMAP-representation of the cDC-subsets from naïve PBS, CTA1-II-DD or CTA1-II-CD103 treated mice is depicted and the cluster or treatment distributions of individual $\mathrm{cDCs}$ is given $(\mathrm{A}, \mathrm{B})$. Distribution of $\mathrm{cDC} 1$ and $\mathrm{cDC} 2$ cells among the analyzed cDCs, according to the UMAP, based on $\mathrm{CDC} 1$ and $\mathrm{cDC} 2$ subset-restricted gene definitions $(\mathrm{C})$. Heat map showing the 25 top DEG in the different clusters of migratory cDCs following immunization (D). Top 20 differentially expressed genes (DEG) in CTA1-exposed (DD- + 
CD103 constructs) as opposed to unimmunized cDC1 (left panels) and cDC2 subsets (right panels) (E).

\section{Figure 8}

Pseudotime trajectory of differentially regulated genes in CTA1-exposed CD103-targeted cDCs reveals plasticity of cDC1 cells and ability to induce Th17 cells

The continued scRNAseq analysis explored the data set using Monocle 3 with which we established a developmental trajectory of the CTA1-exposed cDC1 cells. The data set from Seurat was analyzed in Monocle 3 and depicted in the UMAP plot (A) and as represented after using the gene signatures of $\mathrm{cDC} 1$ and $\mathrm{cDC} 2$ cells, as in Fig.7E(B). We undertook a pseudotime trajectory analysis of the data with starting node in cluster $0(\mathrm{C})$. The distribution of $\mathrm{cDC} 1$ cells in the UMAP following different i.n immunizations was depicted (D). The expression level of a Th17-promoting gene signature (IL-1b, IL-6, Tgfb1, Tgfb2, Tnfsf4(Ox40L), Tnfrsf9(CD137), $I L-12 b(\mathrm{p} 40)$, Timd4, $C d 80, C d 86)$ was employed to follow gene expression from naïve PBS to CTA1-exposed cDC1 cells along the trajectory (E). An extended gene set enrichment analysis (GSEA) using a gene list taken from a publication of human inflammatory DCs, reported to promote Th17 responses, was compared with the list of co-differentially expressed genes found in CTA1-exposed cCD1 cells (Segura et al. 2013) (F). The distribution of gene modules which significantly vary across pseudotime is depicted $(\mathrm{G}-\mathrm{H})$. Selected genes from the modules in $\mathrm{G}$ are represented in the trajectory of cDC1 cells (I). 


\section{Supplementary figure legends}

\section{Supplementary Figure 1:}

\section{Gating strategy}

Flow cytometry gating strategy for defining cDC subsets in freshly isolated mesenteric lymph node (MLN) cells.

\section{Supplementary Figure 2:}

The CTA1-CD103 is superior to the CTA1-DD adjuvant at stimulating antibody responses to admixed proteins

Mice were immunized with a fixed dose $(5 \mu \mathrm{g})$ of NP-CGG or TT antigen and a range of doses of the CTA1-II-CD103 or CTA1-II-DD adjuvants were admixed and given i.n in 3 doses with 10 days apart. The immune responses were determined 8 days after the final immunization in serum and bronchioalveolar lavage (BAL) and values are given as mean $\log _{10}$-titers \pm SD of 5 mice in each group and one representative experiment of three is shown. Statistical significance was calculated using ANOVA with Dunnett's post-test; $\mathrm{p}$-values $* * \mathrm{p}<0.01, * * * \mathrm{p}<0.001, * * * *$ $\mathrm{p}<0.0001$.

\section{Supplementary Figure 3:}

\section{CTA1-II-CD103 stimulated CD4 T cell subset differentiation dominated by Th17 cells}

Wild-type C57B1/6 mice (CD45.2 ${ }^{+}$) were given CFSE-labeled CD45.1 ${ }^{+}$OT-II transgenic T cells by adoptive transfer and subsequently immunized i.n. with equimolar doses of $5 \mu \mathrm{M}$ of OVA, CTA1-II-DD, or CTA1-II-CD103 on the next day. OT II cells from mLN were analyzed on day 4 for in vivo proliferation by assessing CFSE-dilution by flow cytometry (A). 
Differentiation into the different functional subsets was done by phenotypic analysis using labelled antibodies to CXCR5 and PD-1 for Tfh, GATA3 for Th2, Tbx21 and CXCR3 for Th1, Foxp3 for Tregs. Gating was done using FMO to identify the subsets. Distribution of OT II cells into the different subsets was are given as mean $\% \pm$ SD of all OT II cells, as indicated, and these are representative of three independent experiments with 2-6 mice in each group (B). Lymphocytes from the mLN from immunized mice as in A were cultured in triplicates with recall antigen p323 peptide for $96 \mathrm{~h}$, after which supernatants were collected and assessed for cytokine content, as indicated. Cytokine production is given in $\mathrm{pg} / \mathrm{ml} \pm \mathrm{SD}$ for each group (C). Statistical significance was calculated using analysis of variance (ANOVA) with Dunnett's post-test. $* \mathrm{p}<0.05, * * \mathrm{p}<0.01, * * * \mathrm{p}<0.001, * * * * \mathrm{p}<0.0001$. Distribution of the different CD4 T cell subsets of OT II cells following a single i.n immunization as indicated. Values are given as a pie chart representing mean \% of all OT II cells and these are calculated from three independent experiments with 2-6 mice in each group (D).

\section{Supplementary Figure 4:}

\section{CTA1-II-CD103 acting on cDC1 cells in vitro promotes Th17 differentiation}

Highly enriched cDC1 cells from unimmunized mice were cultured together with naïve CFSElabelled OT-II cells (CD45.1+) in a 1/10 ratio and 5 $\mu \mathrm{M}$ of CTA1-II-CD103 fusion protein was added to these cultures as indicated. The frequency of Ror $\gamma \mathrm{t}^{+}$or $\mathrm{Tbet}^{+}$OT-II cells in triplicate cultures was determined on day 3 by flow cytometry using labeled antibodies and mean values $\pm \mathrm{SD}$ from one representative experiment of three giving similar results.

\section{Supplementary Figure 5:}


Gene heat map (A) and gene dot plot (B) for differentially expressed cDC1 and cDC2 associated gene sets for migratory cDC cells. Plot illustrating the gene sequence expression pattern in CD103 vs DD-constructs in $\mathrm{cDC} 1$ and $\mathrm{cDC} 2$ cells, respectively(C upper panels). Top 20 differentially expressed genes (DEG) in CD103- compared to DD-targeted cDC1 and cDC2 cells analyzed $20 \mathrm{~h}$ following an i.n administration of $50 \mu \mathrm{M}$ doses and analyzed, as indicated (C lower panels). Gene signatures identifying Th17 promoting gene functions in cDC1 cells isolated from CTA1-DD/CTA1-CD103 vs PBS-treated mice (D). 


\section{References;}

Agren, L. C., L. Ekman, B. Lowenadler, J. G. Nedrud, and N. Y. Lycke. 1999. 'Adjuvanticity of the cholera toxin A1-based gene fusion protein, CTA1-DD, is critically dependent on the ADP-ribosyltransferase and Ig-binding activity', J Immunol, 162: 2432-40.

Agren, L., M. Norin, N. Lycke, and B. Lowenadler. 1999. 'Hydrophobicity engineering of cholera toxin A1 subunit in the strong adjuvant fusion protein CTA1-DD', Protein Eng, 12: 173-8.

Andersen, B. M., J. Xia, A. L. Epstein, J. R. Ohlfest, W. Chen, B. R. Blazar, C. A. Pennell, and M. R. Olin. 2016. 'Monomeric annexin A2 is an oxygen-regulated toll-like receptor 2 ligand and adjuvant', J Immunother Cancer, 4: 11.

Anderson, D. A., 3rd, C. A. Dutertre, F. Ginhoux, and K. M. Murphy. 2020. 'Genetic models of human and mouse dendritic cell development and function', Nat Rev Immunol.

Anderson, D. A., 3rd, K. M. Murphy, and C. G. Briseno. 2018. 'Development, Diversity, and Function of Dendritic Cells in Mouse and Human', Cold Spring Harb Perspect Biol, 10.

Apte, S. H., A. M. Redmond, P. L. Groves, S. Schussek, D. J. Pattinson, and D. L. Doolan. 2013. 'Subcutaneous cholera toxin exposure induces potent CD103(+) dermal dendritic cell activation and migration', Eur J Immunol, 43: 2707-17.

Becht, E., L. McInnes, J. Healy, C. A. Dutertre, I. W. H. Kwok, L. G. Ng, F. Ginhoux, and E. W. Newell. 2018. 'Dimensionality reduction for visualizing single-cell data using UMAP', Nat Biotechnol.

Bedoui, S., and W. R. Heath. 2015. 'Kruppel-ling of IRF4-Dependent DCs into Two Functionally Distinct DC Subsets', Immunity, 42: 785-7.

Bernasconi, V., K. Norling, M. Bally, F. Hook, and N. Y. Lycke. 2016. 'Mucosal Vaccine Development Based on Liposome Technology', J Immunol Res, 2016: 5482087.

Bernasconi, V., K. Norling, I. Gribonika, L. C. Ong, S. Burazerovic, N. Parveen, K. Schon, A. Stensson, M. Bally, G. Larson, F. Hook, and N. Lycke. 2020. 'A vaccine combination of lipid nanoparticles and a cholera toxin adjuvant derivative greatly improves lung protection against influenza virus infection', Mucosal Immunol.

Bhaumik, S., and R. Basu. 2017. 'Cellular and Molecular Dynamics of Th17 Differentiation and its Developmental Plasticity in the Intestinal Immune Response', Front Immunol, 8: 254 .

Boscardin, S. B., J. C. Hafalla, R. F. Masilamani, A. O. Kamphorst, H. A. Zebroski, U. Rai, A. Morrot, F. Zavala, R. M. Steinman, R. S. Nussenzweig, and M. C. Nussenzweig. 2006. 'Antigen targeting to dendritic cells elicits long-lived T cell help for antibody responses', J Exp Med, 203: 599-606.

Briseno, C. G., A. T. Satpathy, J. T. th Davidson, S. T. Ferris, V. Durai, P. Bagadia, K. W. O'Connor, D. J. Theisen, T. L. Murphy, and K. M. Murphy. 2018. 'Notch2-dependent DC2s mediate splenic germinal center responses', Proc Natl Acad Sci U S A, 115: 10726-31.

Brown, C. C., H. Gudjonson, Y. Pritykin, D. Deep, V. P. Lavallee, A. Mendoza, R. Fromme, L. Mazutis, C. Ariyan, C. Leslie, D. Pe'er, and A. Y. Rudensky. 2019. 'Transcriptional Basis of Mouse and Human Dendritic Cell Heterogeneity', Cell, 179: 846-63 e24.

Cabeza-Cabrerizo, M., J. van Blijswijk, S. Wienert, D. Heim, R. P. Jenkins, P. Chakravarty, N. Rogers, B. Frederico, S. Acton, E. Beerling, J. van Rheenen, H. Clevers, B. U. Schraml, M. Bajenoff, M. Gerner, R. N. Germain, E. Sahai, F. Klauschen, and C. Reis e Sousa. 2019. 'Tissue clonality of dendritic cell subsets and emergency DCpoiesis revealed by multicolor fate mapping of DC progenitors', Sci Immunol, 4.

Cancel, J. C., K. Crozat, M. Dalod, and R. Mattiuz. 2019. 'Are Conventional Type 1 Dendritic Cells Critical for Protective Antitumor Immunity and How?', Front Immunol, 10: 9.

Cao, J., M. Spielmann, X. Qiu, X. Huang, D. M. Ibrahim, A. J. Hill, F. Zhang, S. Mundlos, L. Christiansen, F. J. Steemers, C. Trapnell, and J. Shendure. 2019. 'The single-cell transcriptional landscape of mammalian organogenesis', Nature, 566: 496-502. 
Carmody, R. J., Q. Ruan, H. C. Liou, and Y. H. Chen. 2007. 'Essential roles of c-Rel in TLRinduced IL-23 p19 gene expression in dendritic cells', J Immunol, 178: 186-91.

Castro, G., X. Liu, K. Ngo, A. De Leon-Tabaldo, S. Zhao, R. Luna-Roman, J. Yu, T. Cao, R. Kuhn, P. Wilkinson, K. Herman, M. I. Nelen, J. Blevitt, X. Xue, A. Fourie, and W. P. Fung-Leung. 2017. 'ROR $\gamma t$ and ROR $\alpha$ signature genes in human Th17 cells', PLoS One, 12: e0181868.

Cook, M. E., N. N. Jarjour, C. C. Lin, and B. T. Edelson. 2020. 'Transcription Factor Bhlhe40 in Immunity and Autoimmunity', Trends Immunol, 41: 1023-36.

Cruz, L. J., R. A. Rosalia, J. W. Kleinovink, F. Rueda, C. W. Lowik, and F. Ossendorp. 2014. 'Targeting nanoparticles to CD40, DEC-205 or CD11c molecules on dendritic cells for efficient CD8(+) T cell response: a comparative study', J Control Release, 192: 20918.

Dudziak, D., A. O. Kamphorst, G. F. Heidkamp, V. R. Buchholz, C. Trumpfheller, S. Yamazaki, C. Cheong, K. Liu, H. W. Lee, C. G. Park, R. M. Steinman, and M. C. Nussenzweig. 2007. 'Differential antigen processing by dendritic cell subsets in vivo', Science, 315: 107-11.

Durai, V., and K. M. Murphy. 2016. 'Functions of Murine Dendritic Cells', Immunity, 45: 719-36.

Dutertre, C. A., E. Becht, S. E. Irac, A. Khalilnezhad, V. Narang, S. Khalilnezhad, P. Y. Ng, L. L. van den Hoogen, J. Y. Leong, B. Lee, M. Chevrier, X. M. Zhang, P. J. A. Yong, G. Koh, J. Lum, S. W. Howland, E. Mok, J. Chen, A. Larbi, H. K. K. Tan, T. K. H. Lim, P. Karagianni, A. G. Tzioufas, B. Malleret, J. Brody, S. Albani, J. van Roon, T. Radstake, E. W. Newell, and F. Ginhoux. 2019. 'Single-Cell Analysis of Human Mononuclear Phagocytes Reveals Subset-Defining Markers and Identifies Circulating Inflammatory Dendritic Cells', Immunity, 51: 573-89 e8.

Eliasson, D. G., K. El Bakkouri, K. Schon, A. Ramne, E. Festjens, B. Lowenadler, W. Fiers, X. Saelens, and N. Lycke. 2008. 'CTA1-M2e-DD: a novel mucosal adjuvant targeted influenza vaccine', Vaccine, 26: 1243-52.

Eliasson, D. G., A. Omokanye, K. Schon, U. A. Wenzel, V. Bernasconi, M. Bemark, A. Kolpe, K. El Bakkouri, T. Ysenbaert, L. Deng, W. Fiers, X. Saelens, and N. Lycke. 2018. 'M2e-tetramer-specific memory CD4 T cells are broadly protective against influenza infection', Mucosal Immunol, 11: 273-89.

Ferris, S. T., V. Durai, R. Wu, D. J. Theisen, J. P. Ward, M. D. Bern, J. T. th Davidson, P. Bagadia, T. Liu, C. G. Briseno, L. Li, W. E. Gillanders, G. F. Wu, W. M. Yokoyama, T. L. Murphy, R. D. Schreiber, and K. M. Murphy. 2020. 'cDC1 prime and are licensed by CD4(+) T cells to induce anti-tumour immunity', Nature, 584: 624-29.

Gribonika, I., D. G. Eliasson, R. K. Chandode, K. Schon, A. Stromberg, M. Bemark, and N. Y. Lycke. 2019. 'Class-switch recombination to IgA in the Peyer's patches requires natural thymus-derived Tregs and appears to be antigen independent', Mucosal Immunol, 12: 1268-79.

Guilliams, M., F. Ginhoux, C. Jakubzick, S. H. Naik, N. Onai, B. U. Schraml, E. Segura, R. Tussiwand, and S. Yona. 2014. 'Dendritic cells, monocytes and macrophages: a unified nomenclature based on ontogeny', Nat Rev Immunol, 14: 571-8.

Hartung, E., M. Becker, A. Bachem, N. Reeg, A. Jakel, A. Hutloff, H. Weber, C. Weise, C. Giesecke, V. Henn, S. Gurka, K. Anastassiadis, H. W. Mages, and R. A. Kroczek. 2015. 'Induction of potent CD8 T cell cytotoxicity by specific targeting of antigen to cross-presenting dendritic cells in vivo via murine or human XCR1', J Immunol, 194: 1069-79.

Heath, W. R., and F. R. Carbone. 2009. 'Dendritic cell subsets in primary and secondary T cell responses at body surfaces', Nat Immunol, 10: 1237-44.

Hirota, K., H. Ahlfors, J. H. Duarte, and B. Stockinger. 2012. 'Regulation and function of innate and adaptive interleukin-17-producing cells', EMBO Rep, 13: 113-20.

Lee, J., B. Kim, H. Chu, K. Zhang, H. Kim, J. H. Kim, S. H. Kim, Y. Pan, J. Y. Noh, Z. Sun, J. Lee, K. Y. Jeong, K. H. Park, J. W. Park, T. S. Kupper, C. O. Park, and K. H. Lee. 
2020. 'FABP5 as a possible biomarker in atopic march: FABP5-induced Th17 polarization, both in mouse model and human samples', EBioMedicine, 58: 102879.

Lee, J., J. Zhang, Y. J. Chung, J. H. Kim, C. M. Kook, J. M. Gonzalez-Navajas, D. S. Herdman, B. Nurnberg, P. A. Insel, M. Corr, J. H. Mo, A. Tao, K. Yasuda, I. R. Rifkin, D. H. Broide, R. Sciammas, N. J. Webster, and E. Raz. 2020. 'Inhibition of IRF4 in dendritic cells by PRR-independent and -dependent signals inhibit Th2 and promote Th17 responses', Elife, 9.

Li, B., J. M. Reynolds, R. D. Stout, D. A. Bernlohr, and J. Suttles. 2009. 'Regulation of Th17 differentiation by epidermal fatty acid-binding protein', J Immunol, 182: 7625-33.

Lycke, N. 2012. 'Recent progress in mucosal vaccine development: potential and limitations', Nat Rev Immunol, 12: 592-605.

Lycke, N., and C. Lebrero-Fernandez. 2018. 'ADP-ribosylating enterotoxins as vaccine adjuvants', Curr Opin Pharmacol, 41: 42-51.

Ma, W., J. Lee, D. Backenroth, Y. J. Zhou, E. Bush, P. Sims, K. Liu, and Y. Shen. 2019. 'Single cell RNA-Seq reveals pre-cDCs fate determined by transcription factor combinatorial dose', BMC Mol Cell Biol, 20: 20.

Macri, C., C. Dumont, A. P. Johnston, and J. D. Mintern. 2016. 'Targeting dendritic cells: a promising strategy to improve vaccine effectiveness', Clin Transl Immunology, 5: e66.

Mangan, M. S., J. Vega-Ramos, L. T. Joeckel, A. J. Mitchell, A. Rizzitelli, B. Roediger, D. Kaiserman, W. W. Weninger, J. A. Villadangos, and P. I. Bird. 2017. 'Serpinb9 is a marker of antigen cross-presenting dendritic cells', Mol Immunol, 82: 50-56.

Mashayekhi, M., M. M. Sandau, I. R. Dunay, E. M. Frickel, A. Khan, R. S. Goldszmid, A. Sher, H. L. Ploegh, T. L. Murphy, L. D. Sibley, and K. M. Murphy. 2011. 'CD8alpha(+) dendritic cells are the critical source of interleukin-12 that controls acute infection by Toxoplasma gondii tachyzoites', Immunity, 35: 249-59.

Mayer, J. U., M. Demiri, W. W. Agace, A. S. MacDonald, M. Svensson-Frej, and S. W. Milling. 2017. 'Different populations of CD11b(+) dendritic cells drive Th2 responses in the small intestine and colon', Nat Commun, 8: 15820.

Mittelbrunn, M., M. Yanez-Mo, D. Sancho, A. Ursa, and F. Sanchez-Madrid. 2002. 'Cutting edge: dynamic redistribution of tetraspanin CD81 at the central zone of the immune synapse in both T lymphocytes and APC', J Immunol, 169: 6691-5.

Murphy, T. L., G. E. Grajales-Reyes, X. Wu, R. Tussiwand, C. G. Briseno, A. Iwata, N. M. Kretzer, V. Durai, and K. M. Murphy. 2016. 'Transcriptional Control of Dendritic Cell Development', Annu Rev Immunol, 34: 93-119.

Olvera-Gomez, I., S. E. Hamilton, Z. Xiao, C. P. Guimaraes, H. L. Ploegh, K. A. Hogquist, L. Wang, and S. C. Jameson. 2012. 'Cholera toxin activates nonconventional adjuvant pathways that induce protective CD8 T-cell responses after epicutaneous vaccination', Proc Natl Acad Sci U S A, 109: 2072-7.

Pati, R., M. Shevtsov, and A. Sonawane. 2018. 'Nanoparticle Vaccines Against Infectious Diseases', Front Immunol, 9: 2224.

Perciani, C. T., L. Y. Liu, L. Wood, and S. A. MacParland. 2020. 'Enhancing Immunity with Nanomedicine: Employing Nanoparticles to Harness the Immune System', ACS Nano.

Perkins, D. J., K. Richard, A. M. Hansen, W. Lai, S. Nallar, B. Koller, and S. N. Vogel. 2018. 'Autocrine-paracrine prostaglandin E2 signaling restricts TLR4 internalization and TRIF signaling', Nat Immunol, 19: 1309-18.

Persson, E. K., H. Uronen-Hansson, M. Semmrich, A. Rivollier, K. Hagerbrand, J. Marsal, S. Gudjonsson, U. Hakansson, B. Reizis, K. Kotarsky, and W. W. Agace. 2013. 'IRF4 transcription-factor-dependent CD103(+)CD11b(+) dendritic cells drive mucosal $\mathrm{T}$ helper 17 cell differentiation', Immunity, 38: 958-69.

Satpathy, A. T., C. G. Briseno, J. S. Lee, D. Ng, N. A. Manieri, W. Kc, X. Wu, S. R. Thomas, W. L. Lee, M. Turkoz, K. G. McDonald, M. M. Meredith, C. Song, C. J. Guidos, R. D. Newberry, W. Ouyang, T. L. Murphy, T. S. Stappenbeck, J. L. Gommerman, M. C. Nussenzweig, M. Colonna, R. Kopan, and K. M. Murphy. 2013. 'Notch2-dependent classical dendritic cells orchestrate intestinal immunity to attaching-and-effacing bacterial pathogens', Nat Immunol, 14: 937-48. 
Schaper, F., and A. B. van Spriel. 2018. 'Antitumor Immunity Is Controlled by Tetraspanin Proteins', Front Immunol, 9: 1185.

Schlitzer, A., V. Sivakamasundari, J. Chen, H. R. Sumatoh, J. Schreuder, J. Lum, B. Malleret, S. Zhang, A. Larbi, F. Zolezzi, L. Renia, M. Poidinger, S. Naik, E. W. Newell, P. Robson, and F. Ginhoux. 2015. 'Identification of cDC1- and cDC2-committed DC progenitors reveals early lineage priming at the common DC progenitor stage in the bone marrow', Nat Immunol, 16: 718-28.

Schussek, S., V. Bernasconi, J. Mattsson, U. A. Wenzel, A. Stromberg, I. Gribonika, K. Schon, and N. Y. Lycke. 2020. 'The CTA1-DD adjuvant strongly potentiates follicular dendritic cell function and germinal center formation, which results in improved neonatal immunization', Mucosal Immunol, 13: 545-57.

See, P., C. A. Dutertre, J. Chen, P. Gunther, N. McGovern, S. E. Irac, M. Gunawan, M. Beyer, K. Handler, K. Duan, H. R. B. Sumatoh, N. Ruffin, M. Jouve, E. GeaMallorqui, R. C. M. Hennekam, T. Lim, C. C. Yip, M. Wen, B. Malleret, I. Low, N. B. Shadan, C. F. S. Fen, A. Tay, J. Lum, F. Zolezzi, A. Larbi, M. Poidinger, J. K. Y. Chan, Q. Chen, L. Renia, M. Haniffa, P. Benaroch, A. Schlitzer, J. L. Schultze, E. W. Newell, and F. Ginhoux. 2017. 'Mapping the human DC lineage through the integration of high-dimensional techniques', Science, 356.

Segura, E., M. Touzot, A. Bohineust, A. Cappuccio, G. Chiocchia, A. Hosmalin, M. Dalod, V. Soumelis, and S. Amigorena. 2013. 'Human inflammatory dendritic cells induce Th17 cell differentiation', Immunity, 38: 336-48.

Semmrich, M., M. Plantinga, M. Svensson-Frej, H. Uronen-Hansson, T. Gustafsson, A. M. Mowat, U. Yrlid, B. N. Lambrecht, and W. W. Agace. 2012. 'Directed antigen targeting in vivo identifies a role for CD103+ dendritic cells in both tolerogenic and immunogenic T-cell responses', Mucosal Immunol, 5: 150-60.

Steinman, R. M., G. Kaplan, M. D. Witmer, and Z. A. Cohn. 1979. 'Identification of a novel cell type in peripheral lymphoid organs of mice. V. Purification of spleen dendritic cells, new surface markers, and maintenance in vitro', J Exp Med, 149: 1-16.

Stuart, T., A. Butler, P. Hoffman, C. Hafemeister, E. Papalexi, W. M. Mauck, 3rd, Y. Hao, M. Stoeckius, P. Smibert, and R. Satija. 2019. 'Comprehensive Integration of Single-Cell Data', Cell, 177: 1888-902 e21.

Tacken, P. J., I. J. de Vries, R. Torensma, and C. G. Figdor. 2007. 'Dendritic-cell immunotherapy: from ex vivo loading to in vivo targeting', Nat Rev Immunol, 7: 790802.

Theisen, D. J., J. T. th Davidson, C. G. Briseno, M. Gargaro, E. J. Lauron, Q. Wang, P. Desai, V. Durai, P. Bagadia, J. R. Brickner, W. L. Beatty, H. W. Virgin, W. E. Gillanders, N. Mosammaparast, M. S. Diamond, L. D. Sibley, W. Yokoyama, R. D. Schreiber, T. L. Murphy, and K. M. Murphy. 2018. 'WDFY4 is required for cross-presentation in response to viral and tumor antigens', Science, 362: 694-99.

Theodoridis, A. A., C. Eich, C. G. Figdor, and A. Steinkasserer. 2011. 'Infection of dendritic cells with herpes simplex virus type 1 induces rapid degradation of CYTIP, thereby modulating adhesion and migration', Blood, 118: 107-15.

Tussiwand, R., B. Everts, G. E. Grajales-Reyes, N. M. Kretzer, A. Iwata, J. Bagaitkar, X. Wu, R. Wong, D. A. Anderson, T. L. Murphy, E. J. Pearce, and K. M. Murphy. 2015. 'Klf4 expression in conventional dendritic cells is required for T helper 2 cell responses', Immunity, 42: 916-28.

Vander Lugt, B., J. Riddell, A. A. Khan, J. A. Hackney, J. Lesch, J. DeVoss, M. T. Weirauch, H. Singh, and I. Mellman. 2017. 'Transcriptional determinants of tolerogenic and immunogenic states during dendritic cell maturation', J Cell Biol, 216: 779-92.

Viret, C., X. He, and C. A. Janeway, Jr. 2000. 'On the self-referential nature of naive MHC class II-restricted T cells', J Immunol, 165: 6183-92.

Watford, W. T., D. Li, D. Agnello, L. Durant, K. Yamaoka, Z. J. Yao, H. J. Ahn, T. P. Cheng, S. R. Hofmann, T. Cogliati, A. Chen, B. D. Hissong, M. R. Husa, P. Schwartzberg, J. J. O'Shea, and M. Gadina. 2006. 'Cytohesin binder and regulator (cybr) is not essential for T- and dendritic-cell activation and differentiation', Mol Cell Biol, 26: 6623-32. 
Webb, G. J., G. M. Hirschfield, and P. J. Lane. 2016. 'OX40, OX40L and Autoimmunity: a Comprehensive Review', Clin Rev Allergy Immunol, 50: 312-32.

Welty, N. E., C. Staley, N. Ghilardi, M. J. Sadowsky, B. Z. Igyarto, and D. H. Kaplan. 2013. 'Intestinal lamina propria dendritic cells maintain $\mathrm{T}$ cell homeostasis but do not affect commensalism', J Exp Med, 210: 2011-24.

Weyd, H. 2016. 'More than just innate affairs - on the role of annexins in adaptive immunity', Biol Chem, 397: 1017-29.

Yosef, N., A. K. Shalek, J. T. Gaublomme, H. Jin, Y. Lee, A. Awasthi, C. Wu, K. Karwacz, S. Xiao, M. Jorgolli, D. Gennert, R. Satija, A. Shakya, D. Y. Lu, J. J. Trombetta, M. R. Pillai, P. J. Ratcliffe, M. L. Coleman, M. Bix, D. Tantin, H. Park, V. K. Kuchroo, and A. Regev. 2013. 'Dynamic regulatory network controlling TH17 cell differentiation', Nature, 496: 461-8.

Zhang, Z., W. Zhong, D. Hinrichs, X. Wu, A. Weinberg, M. Hall, D. Spencer, K. Wegmann, and J. T. Rosenbaum. 2010. 'Activation of OX40 augments Th17 cytokine expression and antigen-specific uveitis', Am J Pathol, 177: 2912-20.

Zuidscherwoude, M., K. Worah, A. van der Schaaf, S. I. Buschow, and A. B. van Spriel. 2017. 'Differential expression of tetraspanin superfamily members in dendritic cell subsets', PLoS One, 12: e0184317. 
Figures

\section{Figure 1}

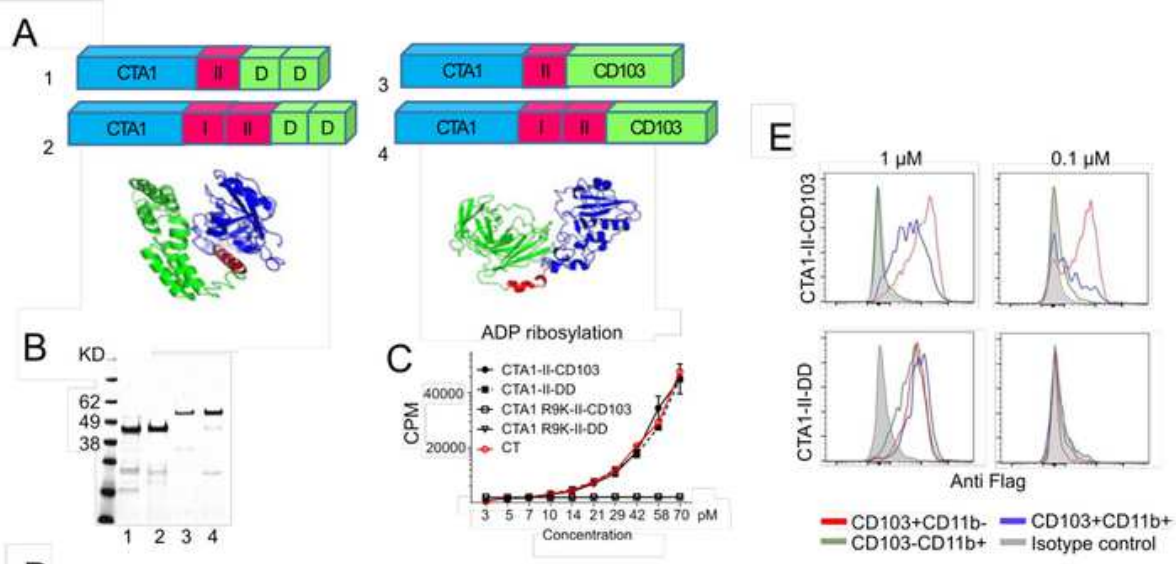

D
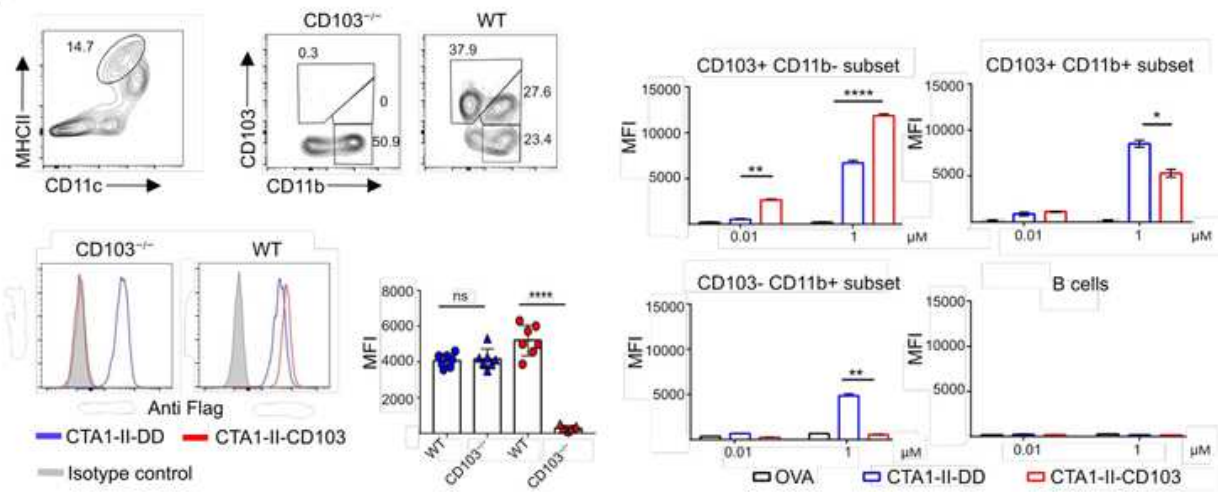

Figure 1

The scFv CD103 fusion protein specifically binds CD103+ DCs populations 


\section{Figure 2}
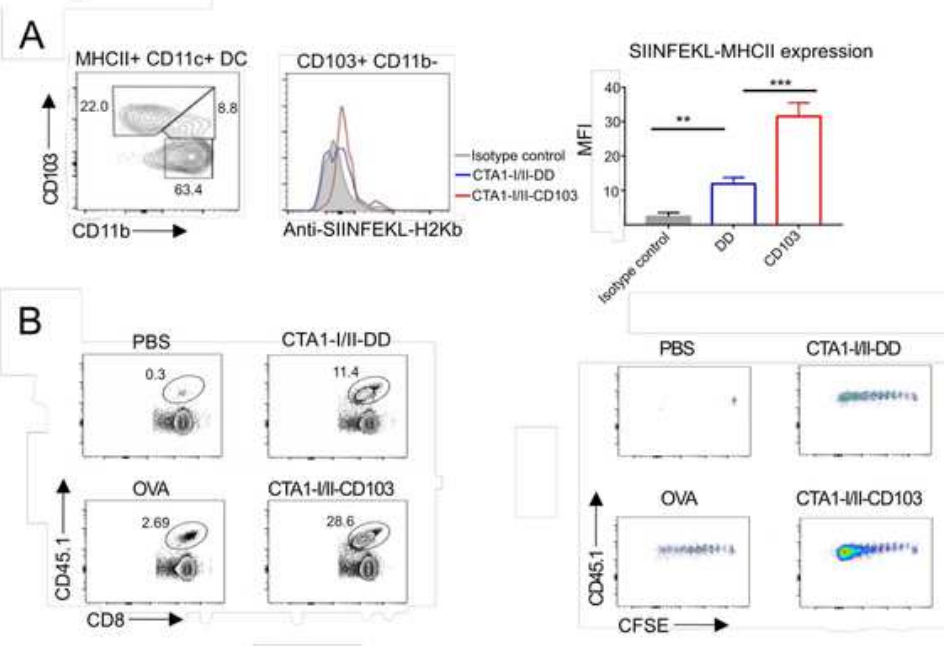

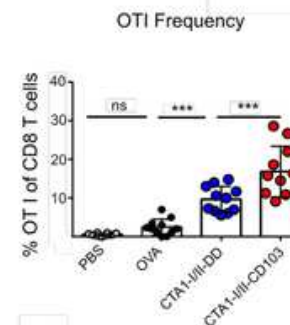

C

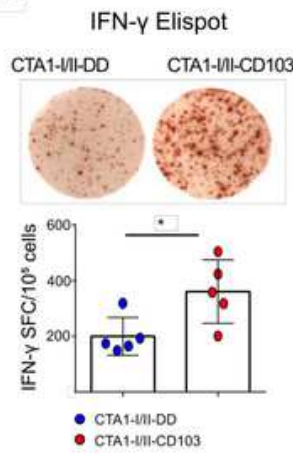

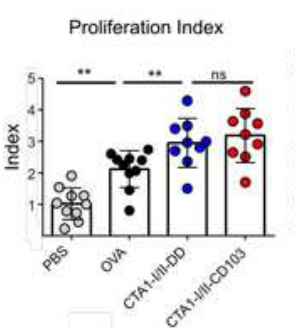

D

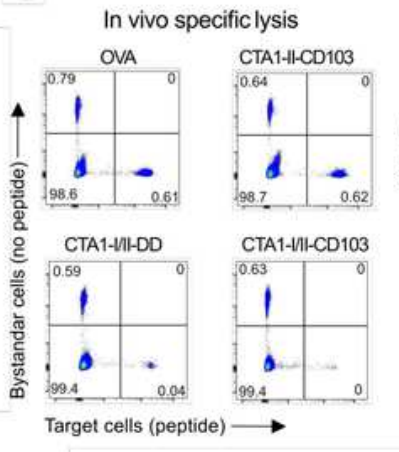

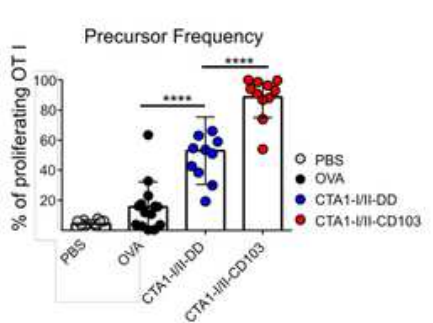

In vivo specific lysis

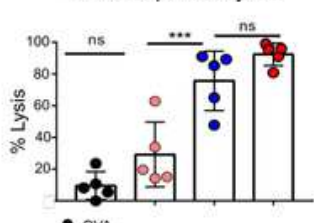

- ova

- CTA1-HI-CD 103

- cTA1-HII-CD103

\section{Figure 2}

The CD103-targeted CTA1-adjuvant acts through cross-presenting CDC1 cells effectively priming CD8 T cells and CTLS 
Figure 3
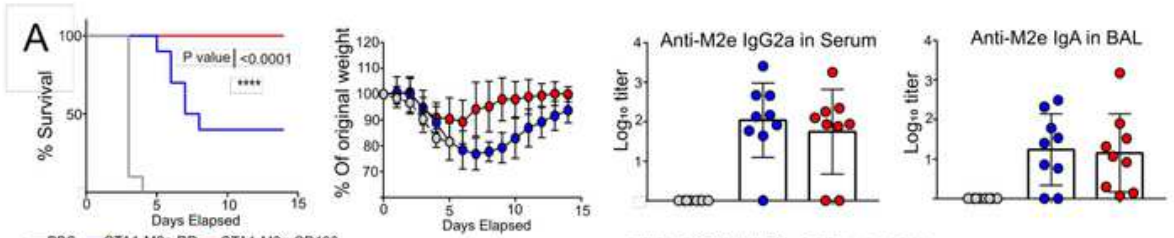

-PBS - CTA1-M2Q-DD - CTA1-M2E-CD103

OPBS -CTA1-M2Q-DD -CTA1-M2O-CD103
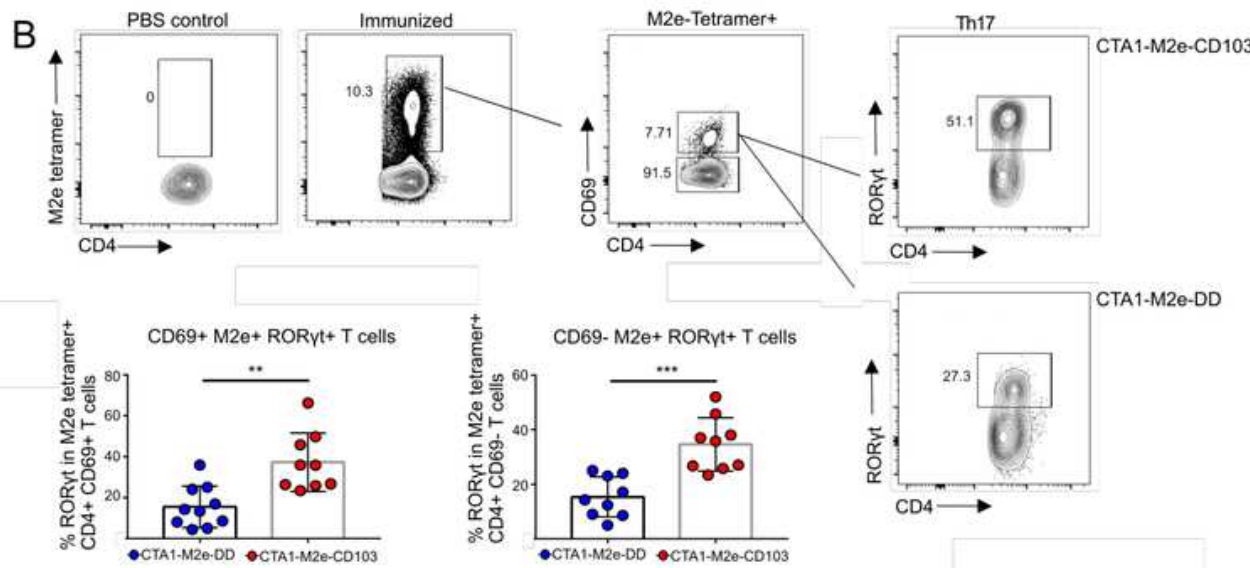

\section{Figure 3}

M2e-tetramer specific CD $4 \mathrm{~T}$ cell protection against influenza virus infection following i.n immunizations with CTA1-M2e-CD103 fusion protein 


\section{Figure 4}

A
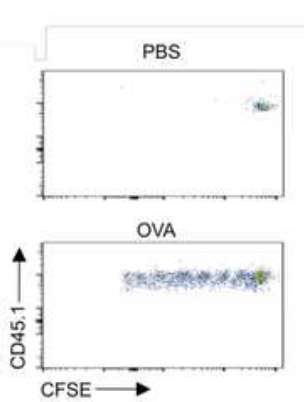

B

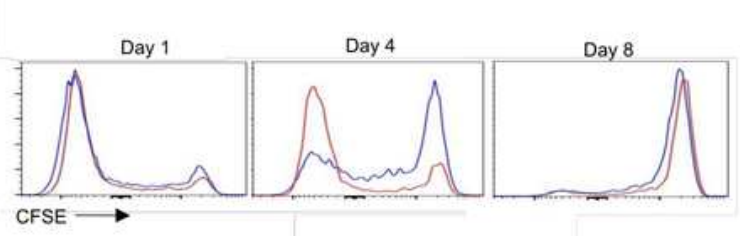

OTII Frequency

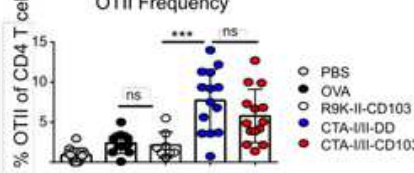

R9K-II-CD103

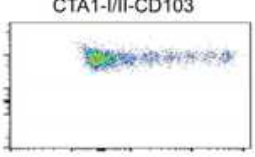

C
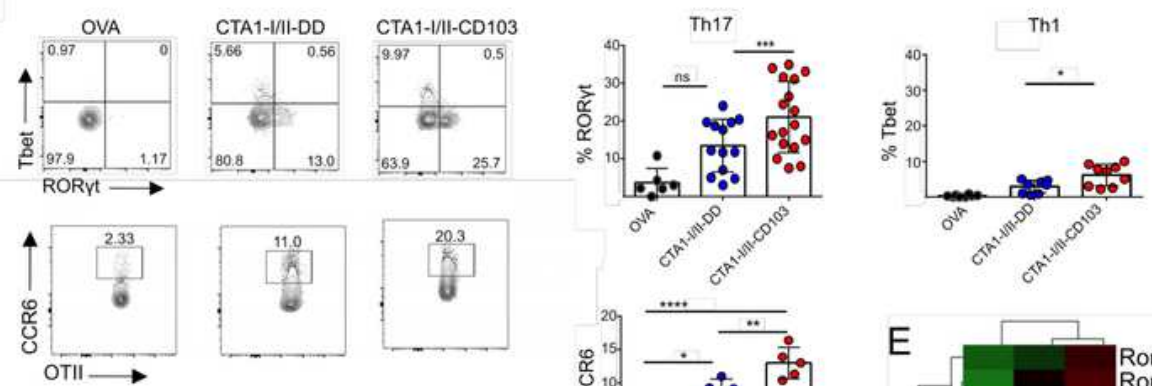

OTII $\longrightarrow$

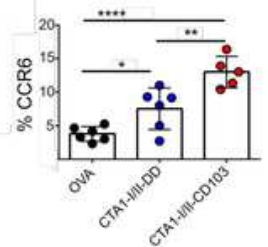

D
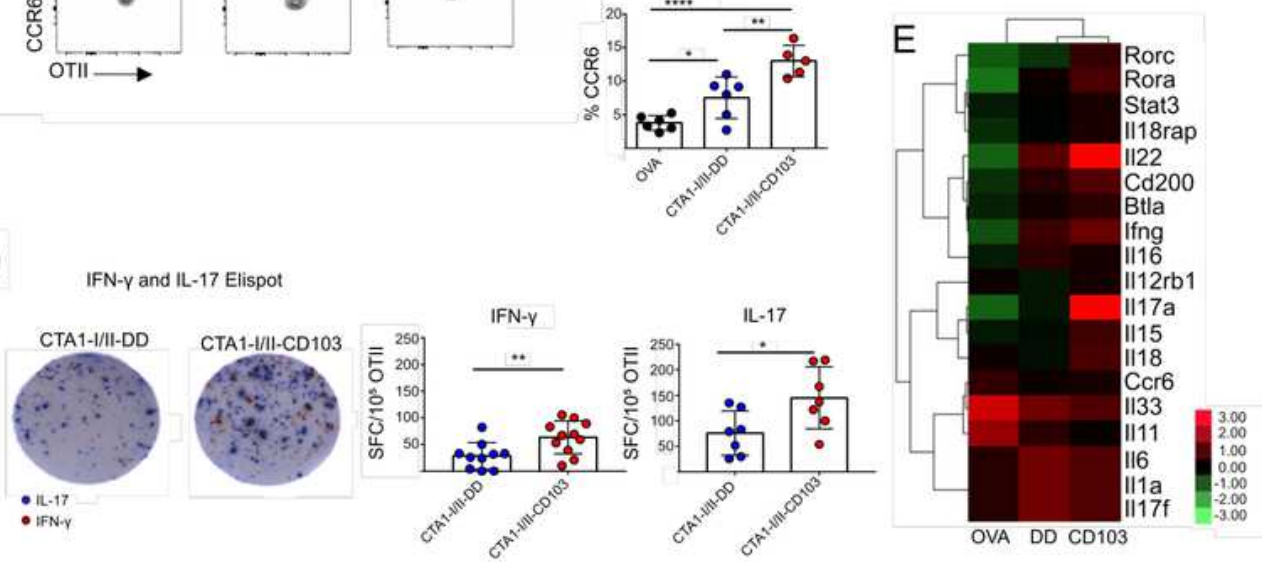

\section{Figure 4}

The CD103-targeted adjuvant effectively primes Th17 cells following i.n immunizations 


\section{Figure 5}

A
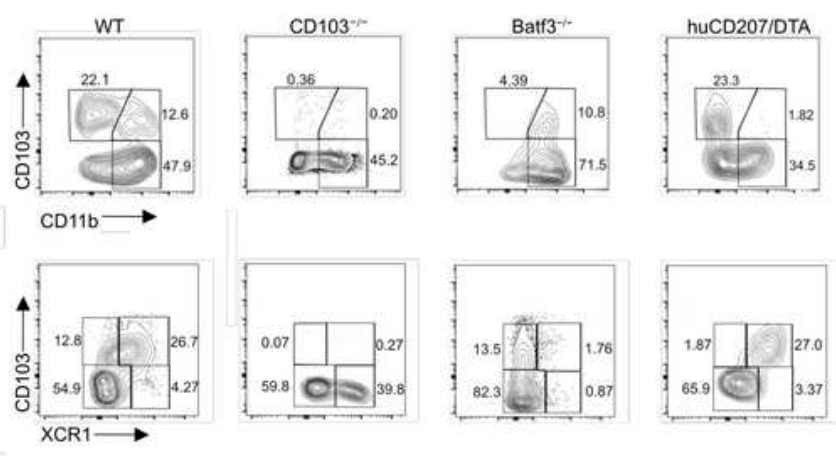

B OTIl cells
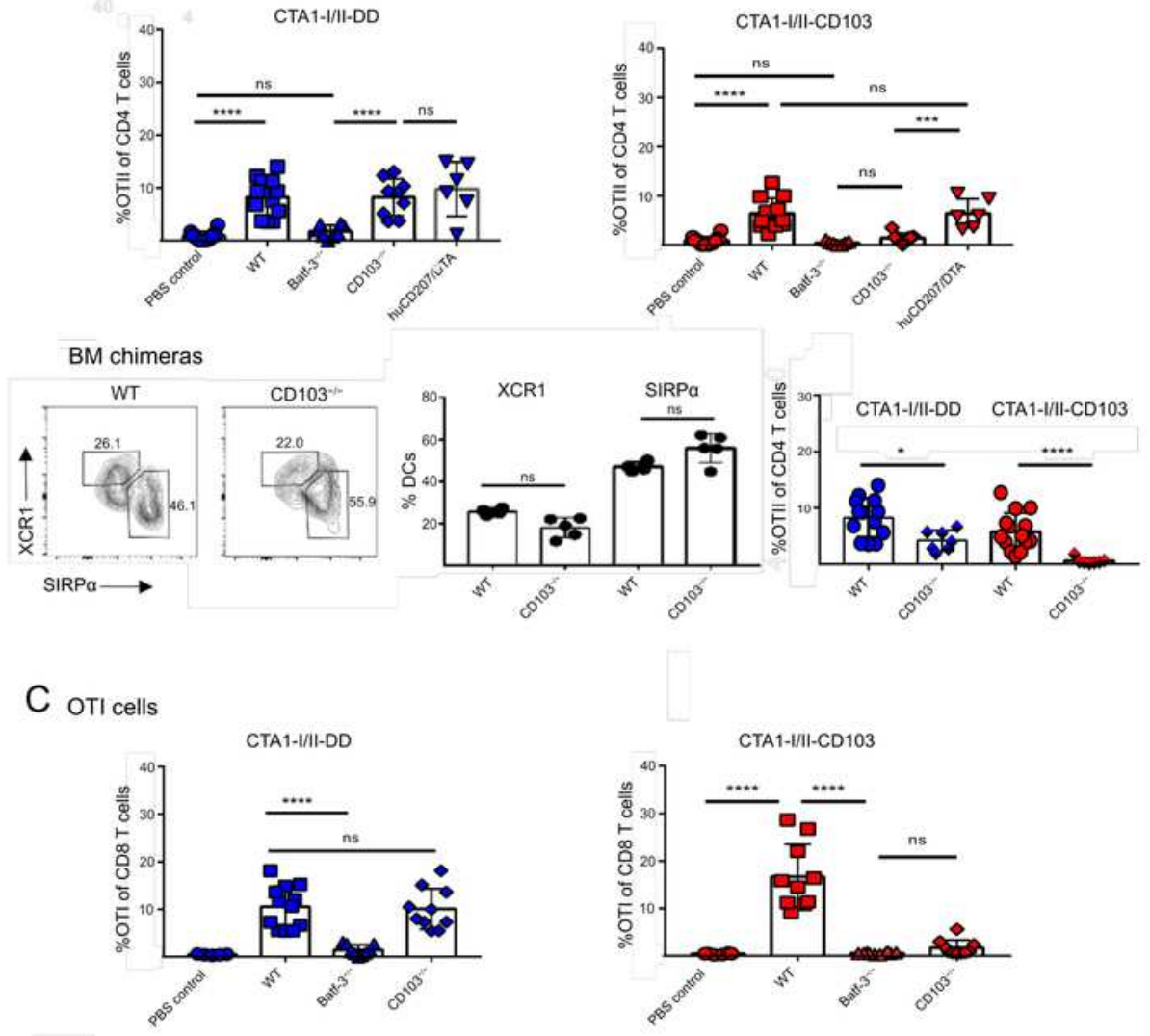

\section{Figure 5}

The CTA1-II-CD103 adjuvant effect is mediated via CDC1 cells 


\section{Figure 6}

\section{A}

In vivo experiment with enriched naive or immunized $\mathrm{CDC} 1$ cells co-cultured with OTII CD4 T cells

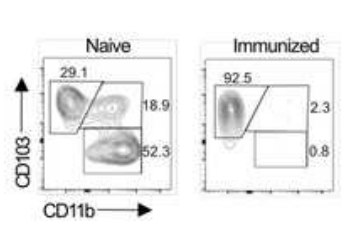

$\mathrm{B}$
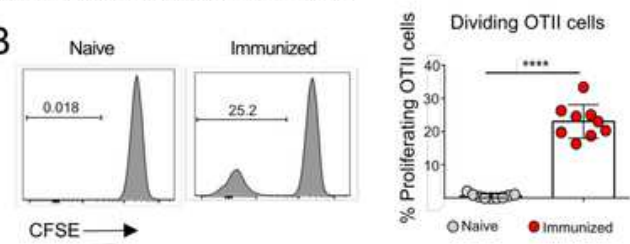

C
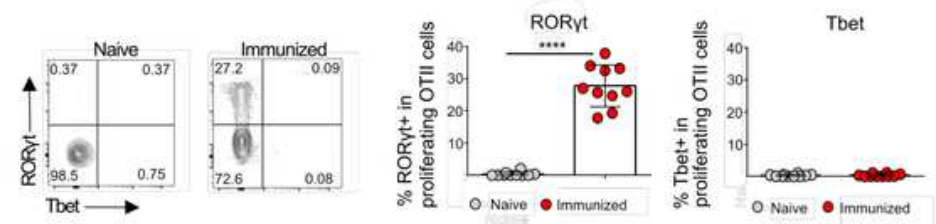

D
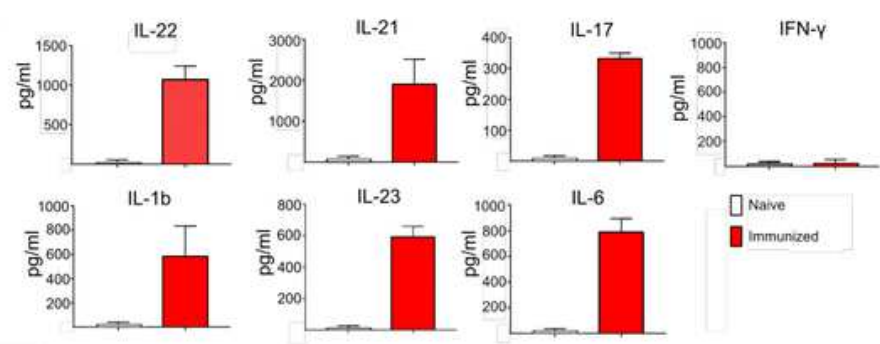

口nave

Dimmunized

E

Different adjuvants; in vitro stimulation of naive Eq-TCR-transgenic CD4 T cells with Eq-peptide and $\mathrm{CDC1}$ cells
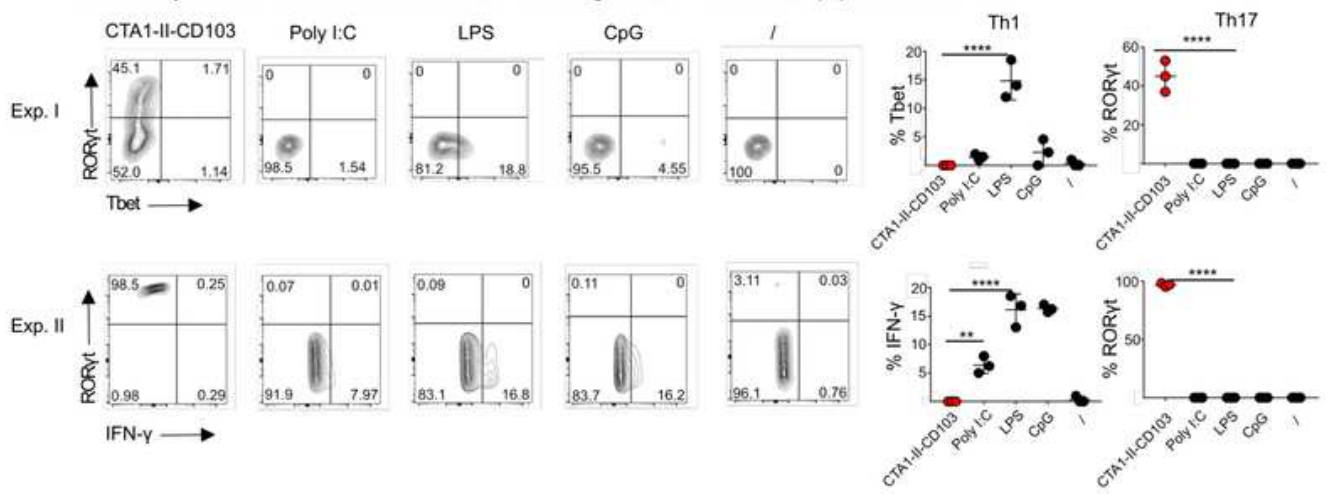

\section{Figure 6}

CTA1-exposed CDC1 cells are potent inducers of Th17 differentiation 
Figure 7
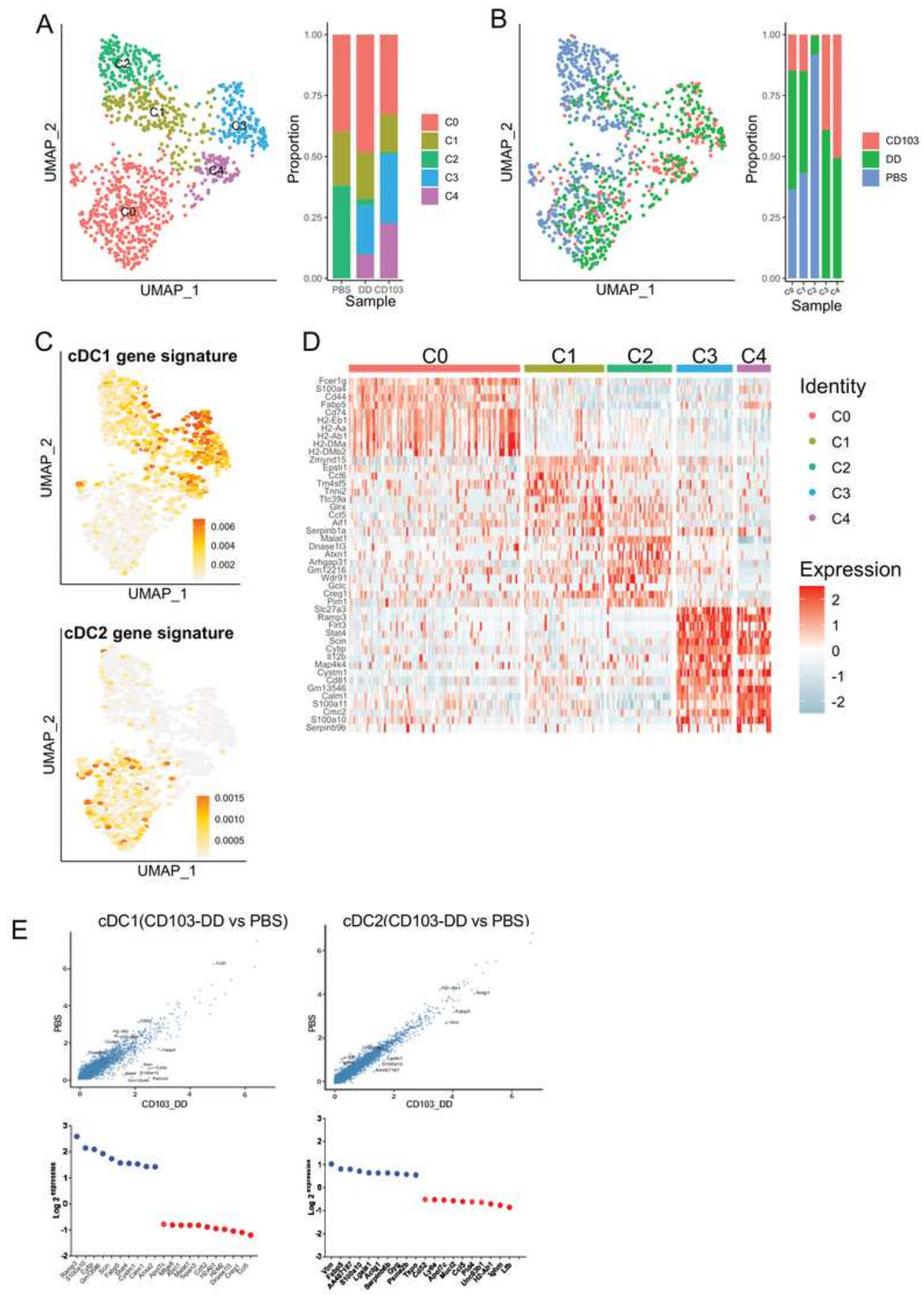

\section{Figure 7}

Single-cell RNAseq analysis of migratory DCs in mLN following i.n immunizations with a CD103-targeted adjuvant 
Figure 8
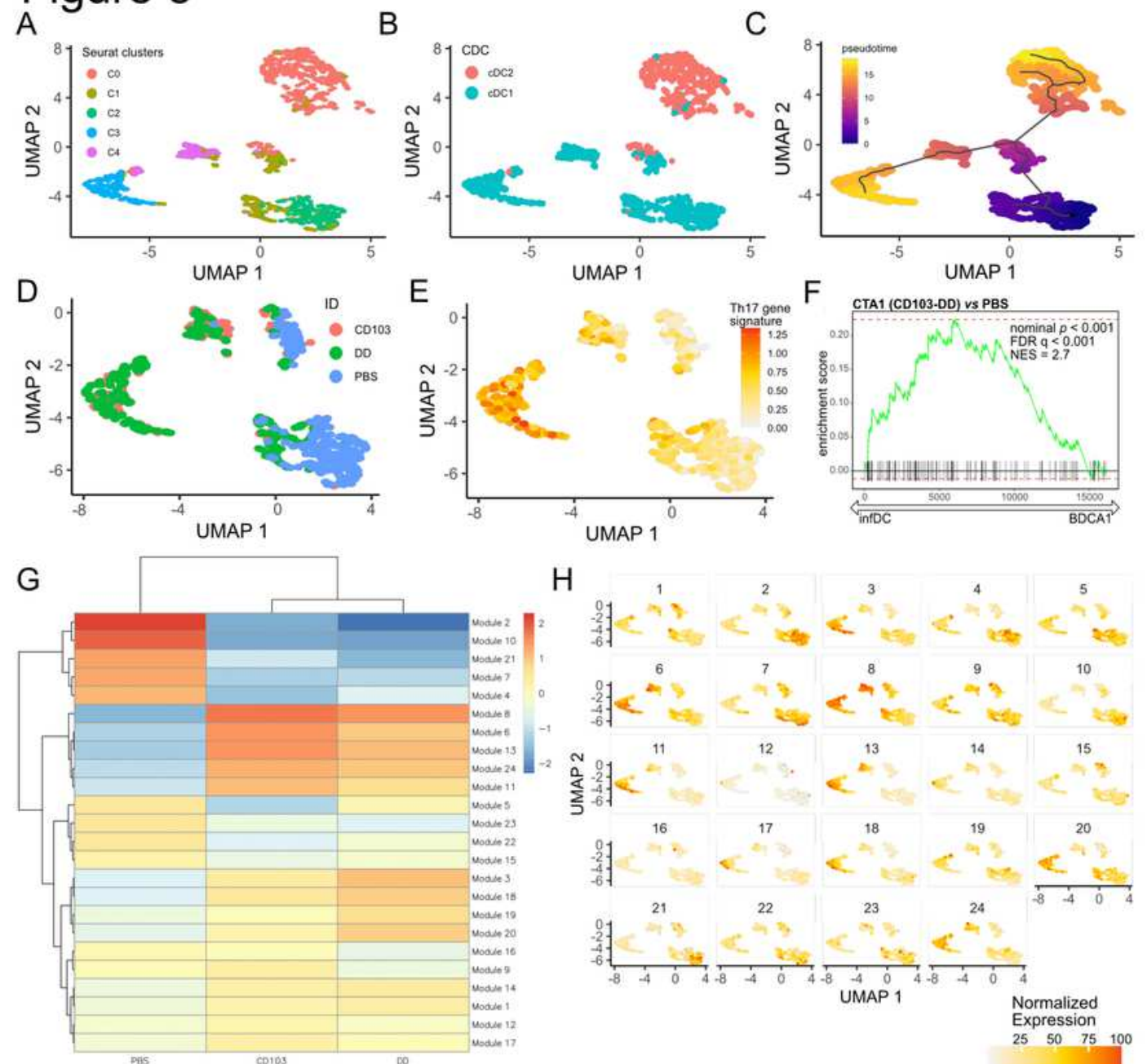

$$
\text { I }
$$

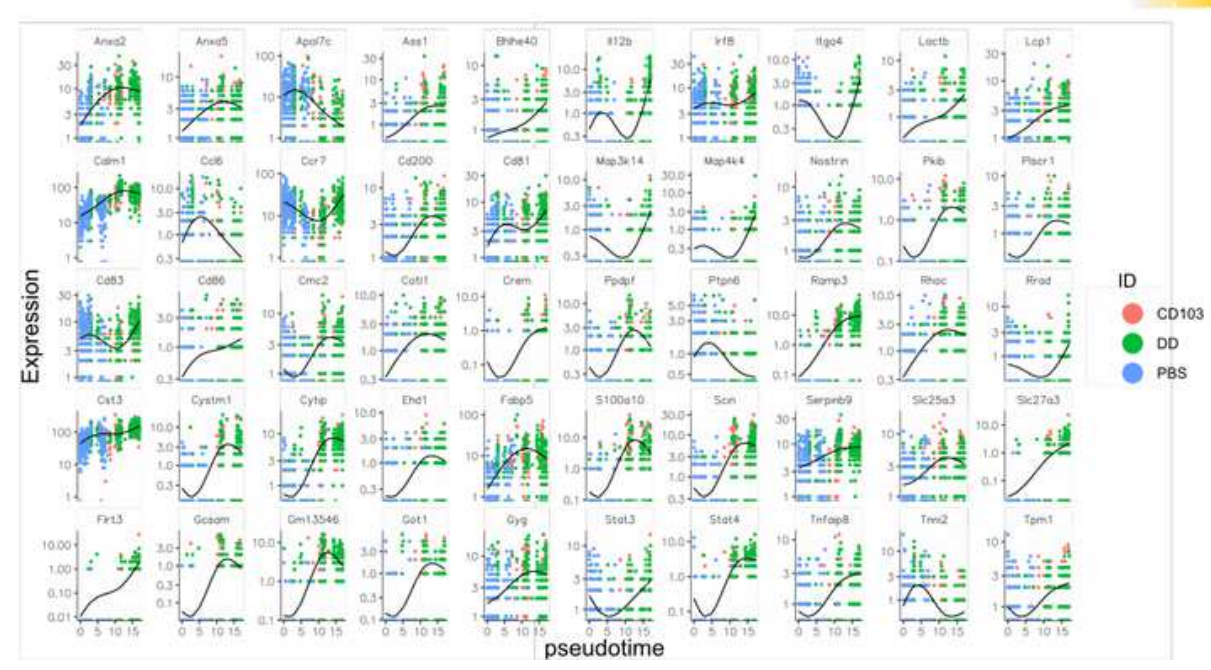

\section{Figure 8}

Pseudotime trajectory of differentially regulated genes in CTA1-exposed CD103-targeted cDCs reveals plasticity of $\mathrm{CDC1}$ cells and ability to induce Th17 cells

\section{Supplementary Files}


This is a list of supplementary files associated with this preprint. Click to download.

- SuplFig5correct1.pdf

- modulesgenes1.xlsx 